\title{
Chemo and Enantioselective Routes to Chiral Fluorinated Hydroxyketones Using Ketoreductases
}

\author{
Brendan T. Grau*, Paul N. Devine, Lisa N. DiMichele, and Birgit \\ Kosjek \\ Department of Process Research, Merck Research Laboratories, Merck and Co. Inc., \\ PO Box 2000, Rahway, NJ, O7065, USA \\ brendan_grau@merck.com
}

Supplemental Information

Sections:

1. Analytical methods

2. Synthesis of methyl/trifluoromethyl diketone substrates $(\mathbf{1}, \mathbf{2}, \mathbf{3})$

3. Analysis of para-diketone $\mathbf{1}$

4. Analysis of meta-diketone 2

5. Analysis of ortho-diketone $\mathbf{3 a}$

6. Synthesis of racemic trifluoromethyl hydroxyketones $(\mathbf{4}, \mathbf{5})$

7. Analysis of para-trifluoromethyl hydroxyketone 4

8. Analysis of meta-trifluoromethyl hydroxyketone $\mathbf{5}$

9. Synthesis of racemic methyl hydroxyketones $(\mathbf{7}, \mathbf{8})$

10. Analysis of para-methyl hydroxyketone 7

11. Analysis of meta-methyl hydroxyketone $\mathbf{8}$

12. Synthesis of bis-alcohols $(\mathbf{1 0}, \mathbf{1 1})$

13. Analysis of para bis-alcohol $\mathbf{1 0}$

14. Analysis of meta bis-alcohol 11 
Section 1: Analytical Methods

RP-HPLC method:

All chemical reactions for synthesis of diketone and product standards were monitored by reverse phase HPLC using a Zorbax Rx-C8 $(4.6 \mathrm{~mm}$ X $25 \mathrm{~cm}, 5 \mathrm{um})$ column at $25 \mathrm{C}$ and a detection wavelength of $210 \mathrm{~nm}$. The mobile phase was $50 \%$ acetonitrile and $50 \%$ $0.1 \% \mathrm{H}_{3} \mathrm{PO}_{4} \mathrm{H}_{2} \mathrm{O}$. Samples were prepared using acetonitrile or acetonitrile: $\mathrm{H}_{2} \mathrm{O}(1: 1)$ as diluent.

Achiral GC method:

All screening reactions of the catalysts were monitored by GC using a Restek Rtx-1701 (30m X 320um, 1um film thickness) column with a split injector (10:1) and a linear velocity of $45 \mathrm{~cm} / \mathrm{sec}$ using helium as the makeup gas. The inlet and detector temperatures were set to $275 \mathrm{C}$ using a FID detector. The compounds were eluted with the following temperature gradient, $200 \mathrm{C}$ hold for 2 minutes, ramp temperature to $275 \mathrm{C}$ at $25 \mathrm{C} / \mathrm{min}$ and hold for $1 \mathrm{~min}$.

Chiral HPLC method:

Reaction samples were extracted with 2 volumes MTBE, dried under $\mathrm{N}_{2}$ purge and resuspended in isopropanol. Chiral separation of the enantiomers of trifluoromethyl hydroxyketones $\mathbf{4}$ and $\mathbf{5}$ was achieved on normal phase HPLC using a Chiralcel OJ-H column with $10 \% \mathrm{MeOH}$ in heptane at $1 \mathrm{~mL} / \mathrm{min}$ and a detection wavelength of $210 \mathrm{~nm}$.

Chiral GC method:

Reaction samples were extracted with 2 volumes MTBE, dried under $\mathrm{N}_{2}$ purge and resuspended in isopropanol. Chiral separation of the enantiomers of methyl hydroxyketones $\mathbf{7}$ and $\mathbf{8}$ was achieved on chiral GC using a Chiralsil-dex CB (25m X $320 \mathrm{um}, 0.25 \mathrm{um}$ film thickness) column with a split injector (10:1) and a linear velocity of $60 \mathrm{~cm} / \mathrm{sec}$ using helium as the makeup gas. The inlet and detector temperatures were set to $200 \mathrm{C}$ using a FID detector. Compound 7 was eluted with an isothermal method at 130C. Compound $\mathbf{8}$ was eluted with an isothermal method at 100C. 
Section 2: Synthesis of methyl/trifluoromethyl diketone substrates

To a solution of bromo-acetophenone $(10 \mathrm{~g})$ in toluene $(70 \mathrm{~mL})$ at $25 \mathrm{C}$ was added ethylene glycol (1.5 equivalents) and a catalytic amount of $p$-toluene sulfonic acid. The solution was refluxed overnight with a Dean-Stark trap. Due to the observed distillation of ethylene glycol, an additional 2 equivalents of the reagent was added in order to form the ketal. The toluene solution was cooled to $25 \mathrm{C}$, washed with brine ( $1 / 2$ volume), dried over $\mathrm{MgSO}_{4}$, and concentrated on a rotary evaporator. The $p$-bromo-ketal $(99 \%$ conversion, $96 \%$ yield) was isolated as a fine white solid while the $m$-bromo-ketal (97\% conversion, 93\% yield) and o-bromo-ketal (98\%conversion, 94\% yield) were yellow oils.

The reaction from the ketal to the diketone was modified from the method in the literature reference ${ }^{2}$. 1.1 equivalents $\mathrm{n}$-hexyl lithium $(2.3 \mathrm{M}$ in hexanes) was added to a solution of bromo-ketal $(10 \mathrm{~g})$ in THF $(100 \mathrm{~mL})$ at $-78 \mathrm{C}$ and aged to completion. Methyl trifluoroacetate (1.2 equivalents) was added to quench and the solution was aged. Upon completion, the solution was warmed to $25 \mathrm{C}$ and the ketal was deprotected by addition of $1 \mathrm{~N} \mathrm{HCl}$. The deprotection of the meta and ortho compounds required reflux overnight. The THF solution was extracted into t-butyl methyl ether (MTBE, 2 vol.) and the organic layer was washed with brine ( $1 \mathrm{vol}$.), dried over $\mathrm{MgSO}_{4}$ and concentrated to a crude waxy solid on the rotary evaporator. The crude solids were dissolved in ethyl acetate (EtOAc) and the $p$-diketone $\mathbf{1}$ (65\% yield), $m$-diketone $\mathbf{2}$ (58\% yield), and $o$-diketone $\mathbf{3}$ ( $80 \%$ yield) crystallized as a fine white solid upon addition of heptane. 
Section 3: para-diketone 1

1: $p$-diketone<smiles>CC(=O)c1ccc(C(=O)C(F)(F)F)cc1</smiles>

1: diketone form

1: mono-hydrate form

Para-diketone 1

(mono-hydrate form): ${ }^{1} \mathrm{H}$ NMR $\left(500.13 \mathrm{MHz}, \mathrm{CD}_{3} \mathrm{CN}\right) \delta=8.00(\mathrm{~m}, 2 \mathrm{H}), 7.78(\mathrm{~m}, 2 \mathrm{H})$, $5.40(\mathrm{~s}, 2 \mathrm{H}), 2.59$ (s, 3H) ppm. ${ }^{13} \mathrm{C} \mathrm{NMR}\left(500.13 \mathrm{MHz}, \mathrm{CD}_{3} \mathrm{CN}\right) \delta=198.78,142.83$, 139.20, 128.92, 128.68, 124.19 (q, $\mathrm{J}_{\mathrm{CF}}=287.3 \mathrm{~Hz}$ ), 94.13 (q, $\left.\mathrm{J}_{\mathrm{CF}}=32.0 \mathrm{~Hz}\right), 27.24 \mathrm{ppm}$. ${ }^{19} \mathrm{~F}$ NMR (500.13 MHz, CD $\left.3 \mathrm{CN}\right) \delta=-85.29 \mathrm{ppm}$.

(diketone form): ${ }^{1} \mathrm{H}$ NMR (500.13 MHz, $\left.\mathrm{CD}_{3} \mathrm{CN}\right) \delta=8.18(\mathrm{~m}, 2 \mathrm{H}), 8.13(\mathrm{~m}, 2 \mathrm{H}), 2.64$ (s, $3 \mathrm{H}) \mathrm{ppm} .{ }^{19} \mathrm{~F}$ NMR $\left(500.13 \mathrm{MHz}, \mathrm{CD}_{3} \mathrm{CN}\right) \delta=-73.40 \mathrm{ppm}$.

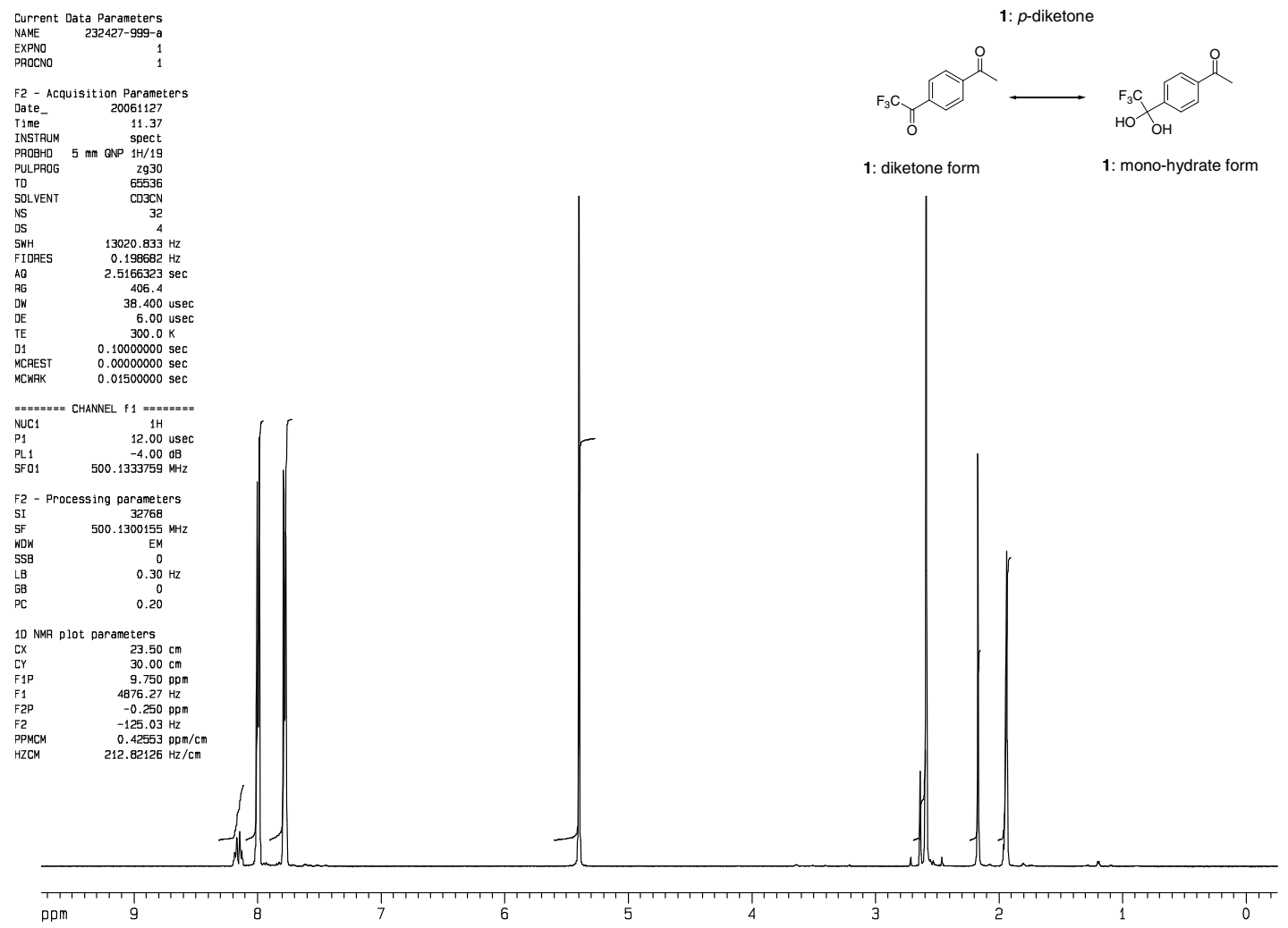



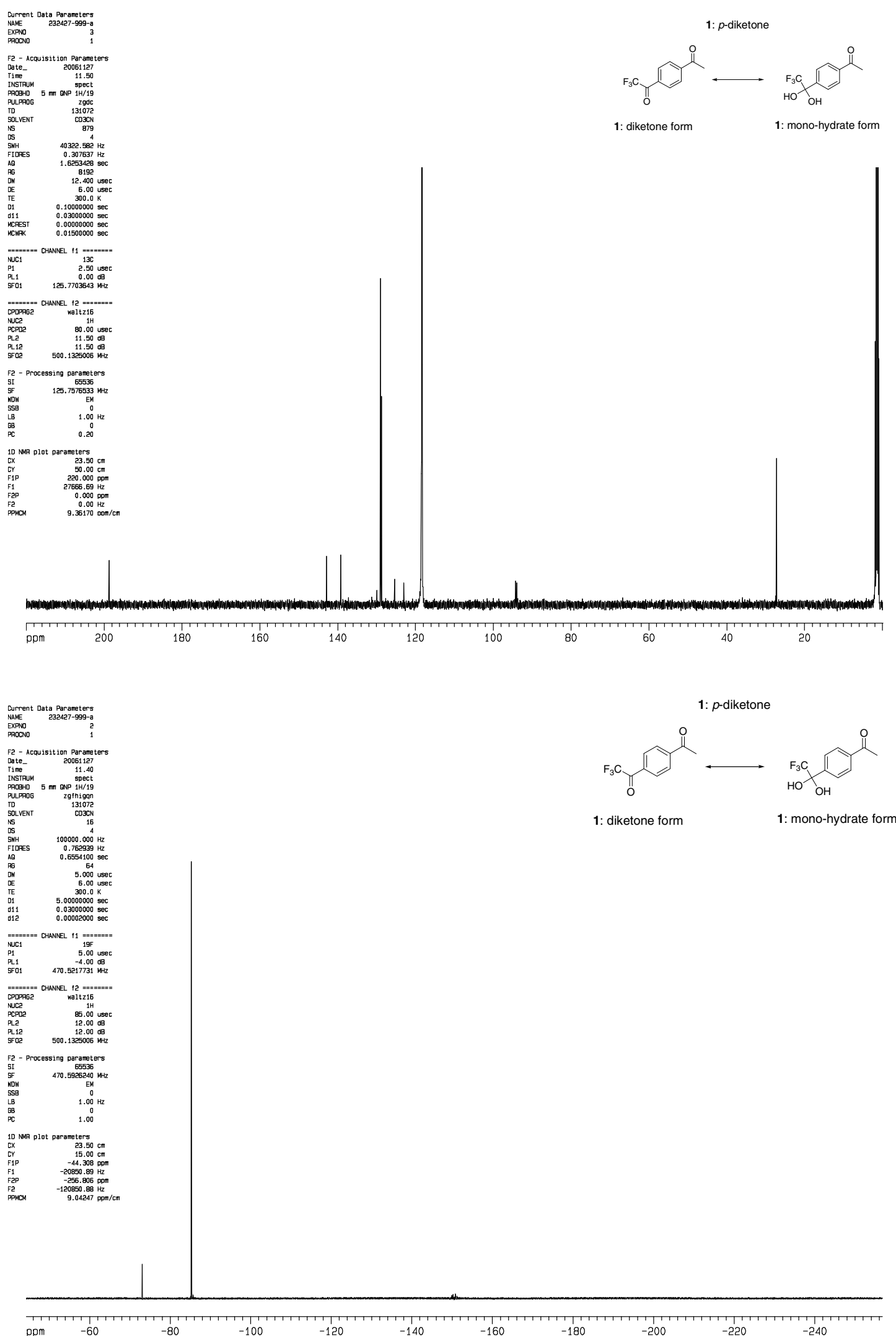
Achiral GC Method:

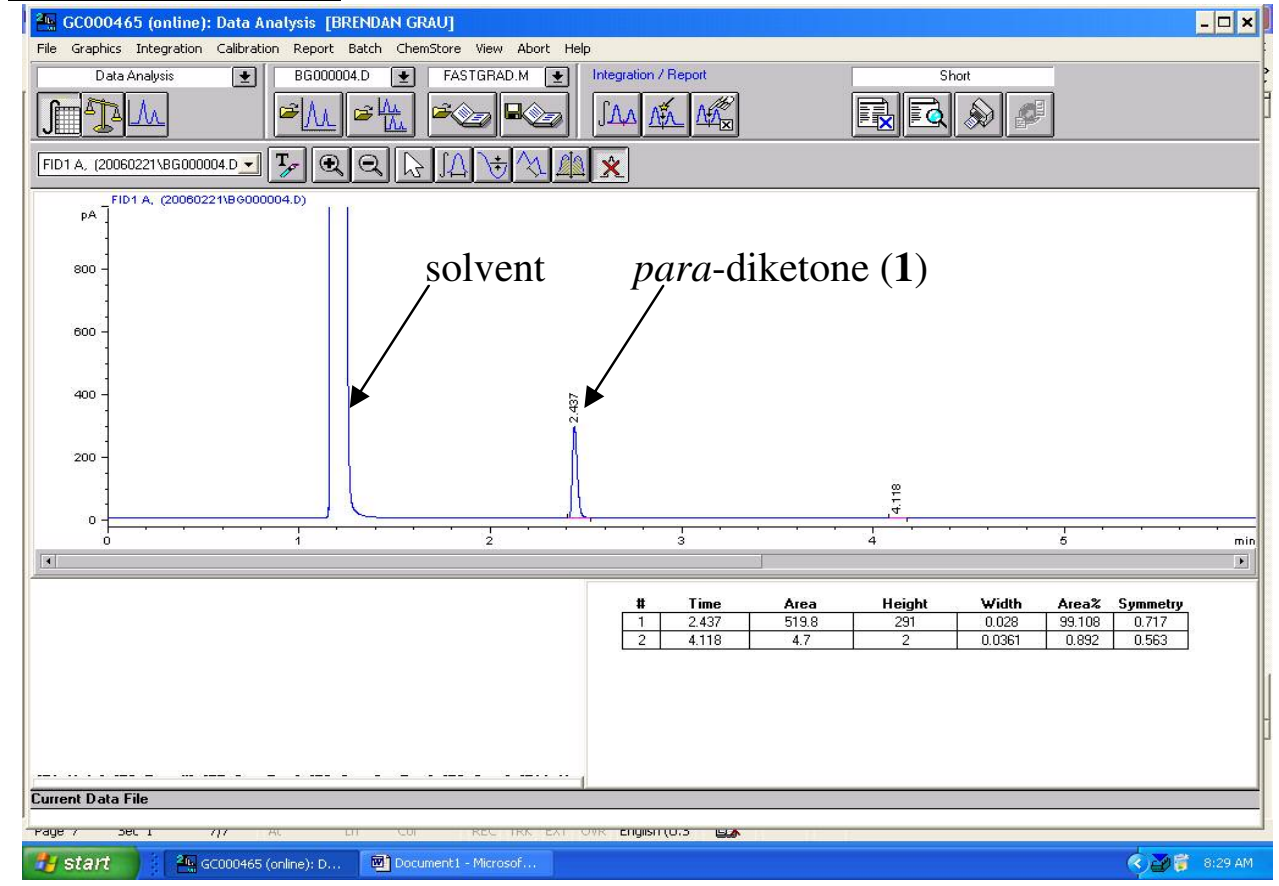


Section 4: meta-diketone 2

\section{2: $m$-diketone}<smiles>CC(=O)c1cccc(C(=O)C(F)(F)F)c1</smiles>

2: diketone form<smiles>CC(=O)c1cccc(C(O)(O)C(F)(F)F)c1</smiles>

2: mono-hydrate form

\section{Meta-diketone 2}

(mono-hydrate form): ${ }^{1} \mathrm{H}$ NMR $\left(500.13 \mathrm{MHz}, \mathrm{CD}_{3} \mathrm{CN}\right) \delta=8.21(\mathrm{~s}, 1 \mathrm{H}), 8.03(\mathrm{~d}, \mathrm{~J}=7.95$ $\mathrm{Hz}, 1 \mathrm{H}), 7.89$ (d, J = 7.95 Hz, 1H), 7.57 (t, J = 7.95 Hz, 1H), 5.43 (s, 2H), 2.59 (s, 3H) ppm. ${ }^{13} \mathrm{C}$ NMR $\left(500.13 \mathrm{MHz}, \mathrm{CD}_{3} \mathrm{CN}\right) \delta=198.71,139.03,138.20,132.82,130.56$, 129.62, 127.83, 124.26 (q, $\left.\mathrm{J}_{\mathrm{CF}}=286.7 \mathrm{~Hz}\right), 94.09\left(\mathrm{q}, \mathrm{J}_{\mathrm{CF}}=32.0 \mathrm{~Hz}\right), 27.17 \mathrm{ppm} .{ }^{19} \mathrm{~F}$ NMR (500.13 MHz, $\left.\mathrm{CD}_{3} \mathrm{CN}\right) \delta=-85.44 \mathrm{ppm}$.

(diketone form): ${ }^{1} \mathrm{H}$ NMR (500.13 MHz, $\left.\mathrm{CD}_{3} \mathrm{CN}\right) \delta=8.56(\mathrm{~s}, 1 \mathrm{H}), 8.35$ (d, J = $7.95 \mathrm{~Hz}$, $1 \mathrm{H}), 8.27(\mathrm{~d}, \mathrm{~J}=7.95 \mathrm{~Hz}, 1 \mathrm{H}), 7.76(\mathrm{t}, \mathrm{J}=7.95 \mathrm{~Hz}, 1 \mathrm{H}), 2.64(\mathrm{~s}, 3 \mathrm{H}) \mathrm{ppm} .{ }^{19} \mathrm{~F} \mathrm{NMR}$ $\left(500.13 \mathrm{MHz}, \mathrm{CD}_{3} \mathrm{CN}\right) \delta=-72.97 \mathrm{ppm}$.

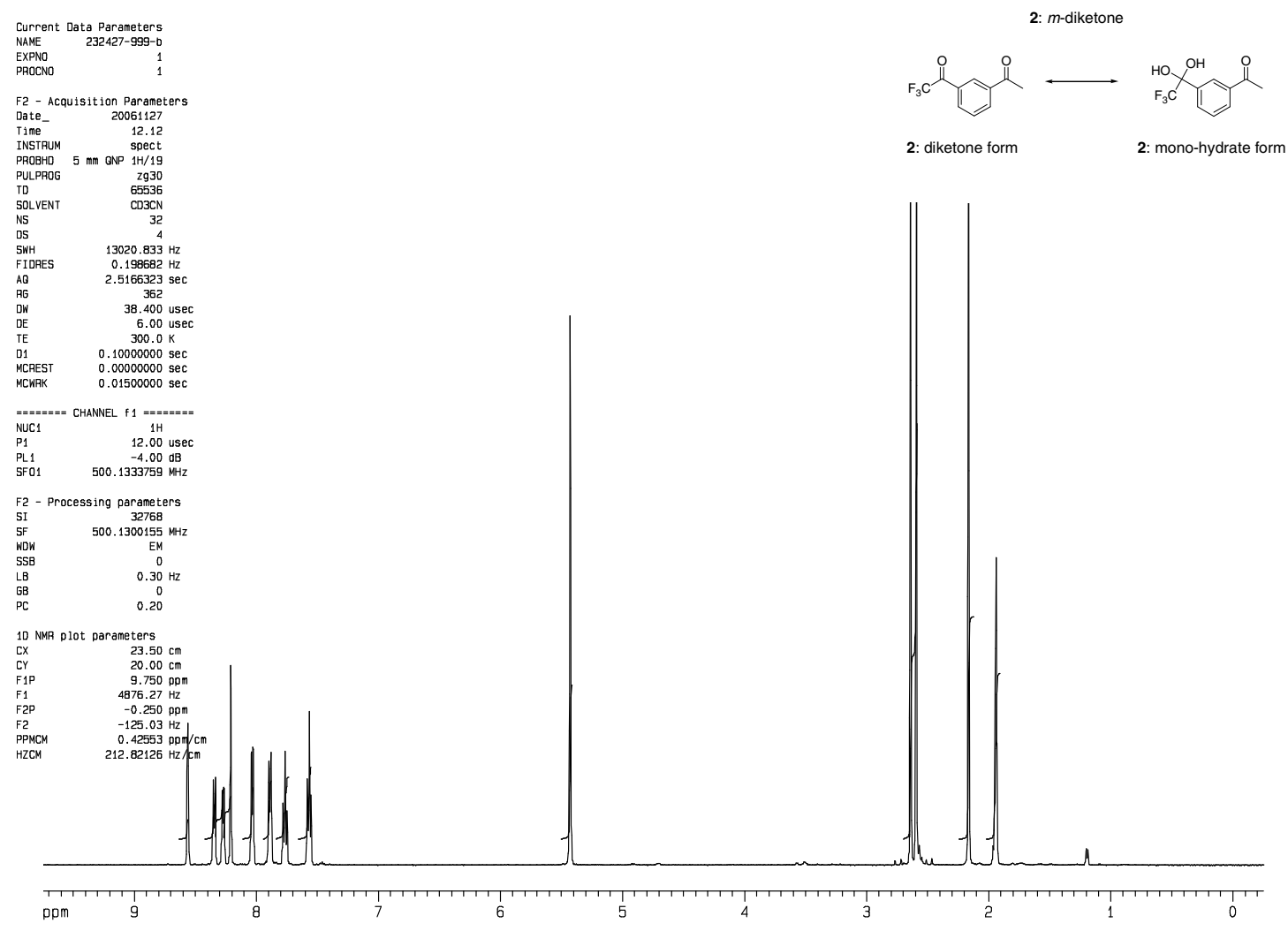



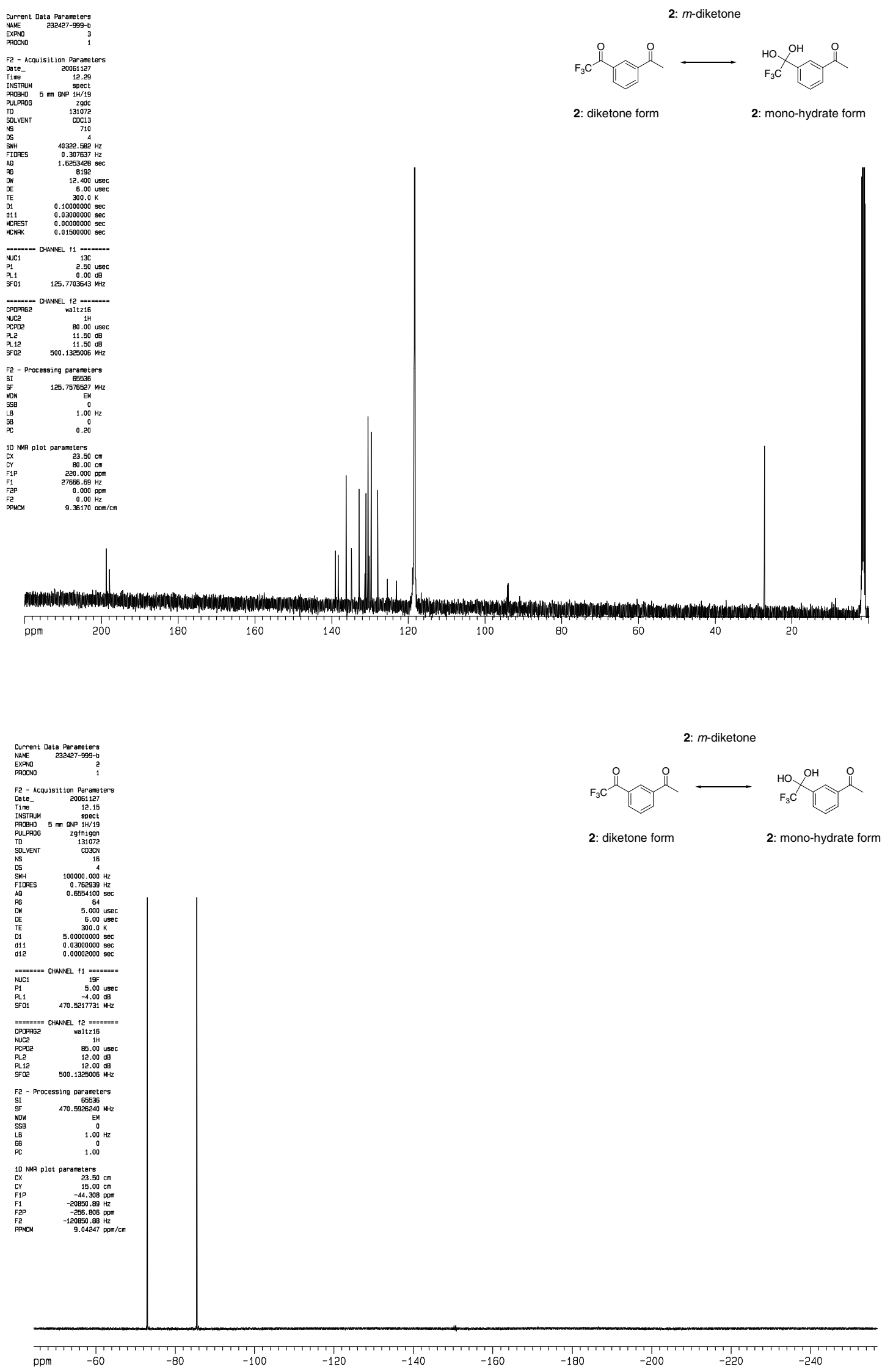
Achiral GC Method:

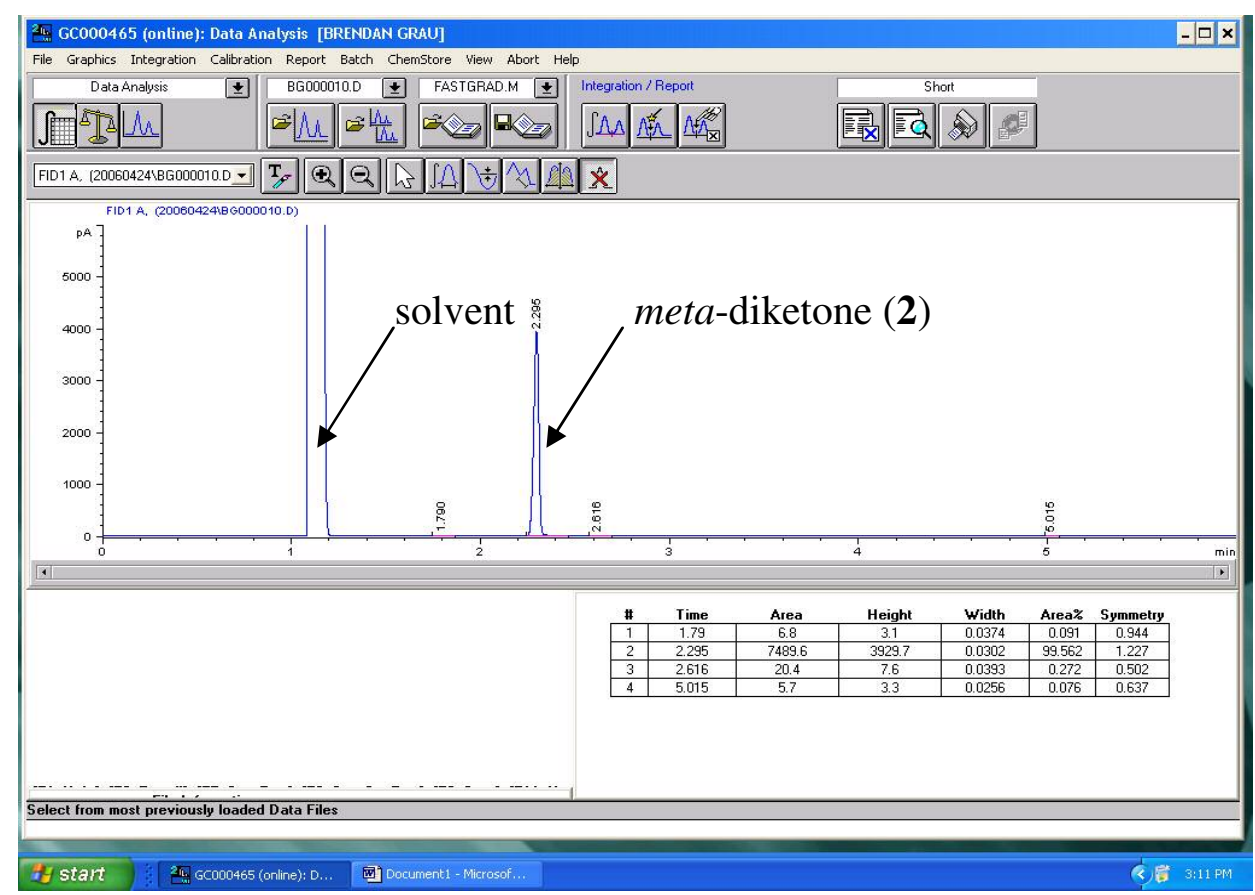


Section 5: ortho-diketone 3a

3a: o-diketone (dihydrate form)<smiles>CC1(O)OC(O)(C(F)(F)F)c2ccccc21</smiles>

Ortho-diketone 3a

(cyclized di-hydrate form): ${ }^{1} \mathrm{H}$ NMR (400.13 MHz, $\left.\mathrm{CD}_{3} \mathrm{CN}\right) \delta=$ ): ${ }^{1} \mathrm{H}$ NMR (500.13 $\left.\mathrm{MHz}, \mathrm{CD}_{3} \mathrm{CN}\right) \delta=[7.60-7.56$ (om, 1H), 7.53-7.50 (om, 2H), 7.47-7.60 (om, 1H), 5.37 (brs, 1H), $4.62(\mathrm{~s}, 1 \mathrm{H}), 1.76(\mathrm{~s}, 3 \mathrm{H})$ and 7.60-7.56 (om, 1H), 7.53-7.50 (om, 2H), 7.477.60 (om, 1H), 5.37 (brs, $1 \mathrm{H}), 4.73$ (brs, $1 \mathrm{H}), 1.70$ (s, 3H) ppm. ${ }^{13} \mathrm{C}$ NMR $(500.13 \mathrm{MHz}$, $\left.\mathrm{CD}_{3} \mathrm{CN}\right) \delta=\left[145.17,135.85,132.41,130.94,124.10\left(\mathrm{q}, \mathrm{J}_{\mathrm{CF}}=286.0 \mathrm{~Hz}\right), 123.34,109.26\right.$, $102.58\left(\mathrm{q}, \mathrm{J}_{\mathrm{CF}}=33.6 \mathrm{~Hz}\right), 27.31 \mathrm{ppm} .{ }^{19} \mathrm{~F} \mathrm{NMR}\left(500.13 \mathrm{MHz}, \mathrm{CD}_{3} \mathrm{CN}\right) \delta=[-84.37$ and 84.15] ppm.

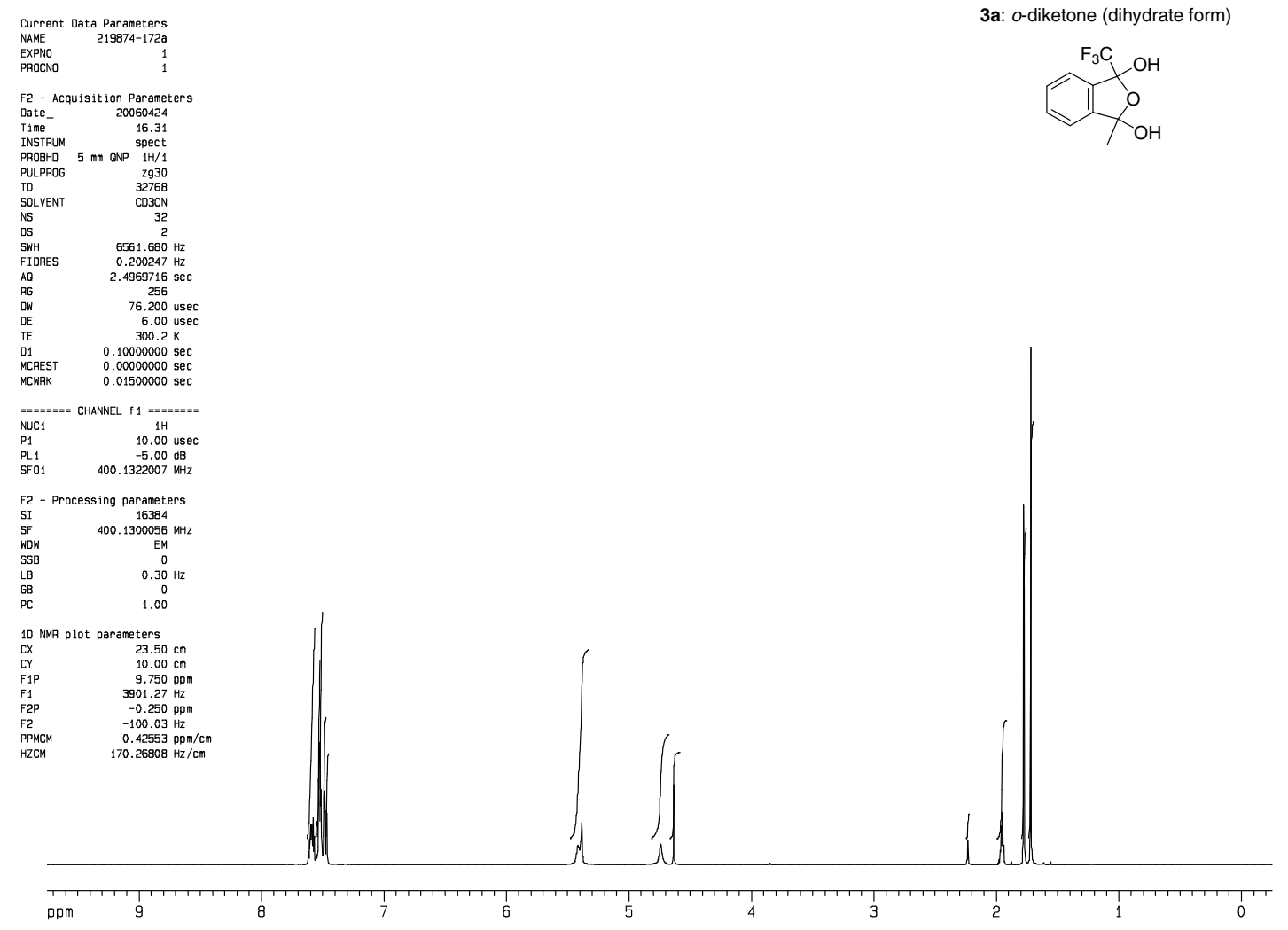




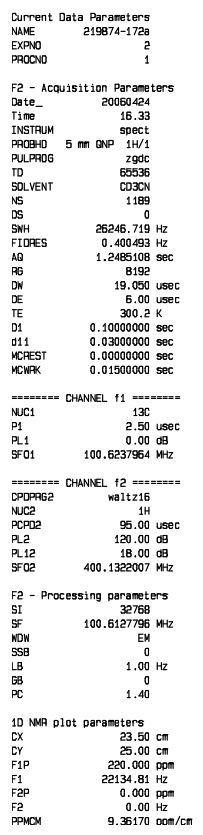

3a: o-diketone (dihydrate form)

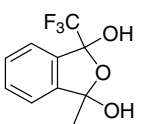

$\quad 9.0 .00 \mathrm{~Hz} / \mathrm{mon}$

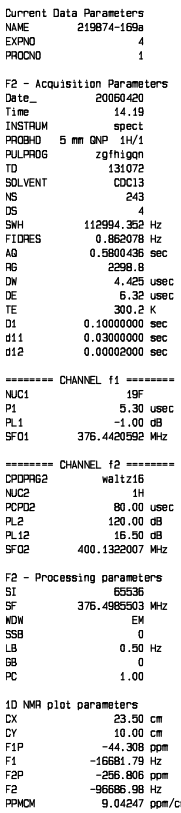

3a: o-diketone (dihydrate form)
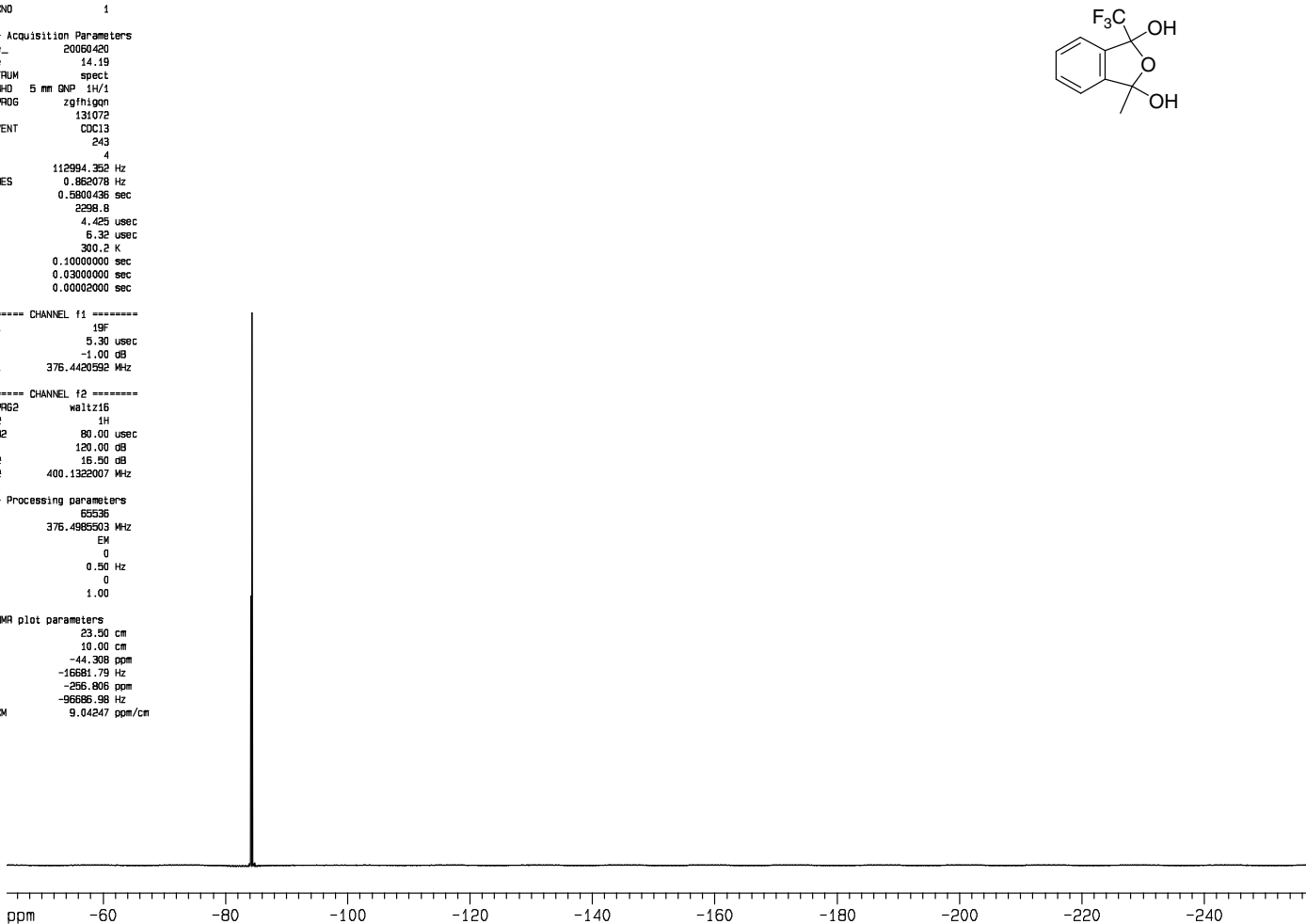
Section 6: Synthesis of racemic trifluoromethyl hydroxyketones $\mathbf{4}$ and $\mathbf{5}$.

To a solution of diketone $\mathbf{1}, \mathbf{2}$, and $\mathbf{3 a}(1 \mathrm{~g})$ in toluene $(20 \mathrm{~mL})$ was added ethylene glycol (4 equivalents) and a catalytic amount of $p$-toluene sulfonic acid. The solution was refluxed overnight with a Dean-Stark to afford the ketal. No reaction was observed on compound 3a. The toluene solution was cooled to $25 \mathrm{C}$ and washed with brine $(1 / 2$ vol.). The organic layer was dried over $\mathrm{MgSO}_{4}$ and concentrated on a rotary evaporator to a crude the crude ketal.

Sodium borohydride (1 equivalent) was added to a solution of ketal ( $1 \mathrm{~g})$ in THF: $\mathrm{H}_{2} \mathrm{O}(10: 1)$ at $25 \mathrm{C}$ and aged $0.5 \mathrm{hr}$. Upon completion the solution was quenched with $1 \mathrm{~N} \mathrm{HCl}$ and aged. The THF solution was extracted into MTBE (2 vol.). The organic layer was washed with brine (1 vol.), dried over $\mathrm{MgSO}_{4}$ and concentrated on a rotary evaporator to yield the crude racemic trifluoromethyl hydroxyketone $\mathbf{4}$ and $\mathbf{5}$. 
Section 7: para-trifluoromethyl hydroxyketone 4 4: $p$-trifluoromethyl hydroxyketone<smiles>CC(=O)c1ccc(C(O)C(F)(F)F)cc1</smiles>

4: ${ }^{1} \mathrm{H}$ NMR $\left(500.13 \mathrm{MHz}, \mathrm{CD}_{3} \mathrm{CN}\right) \delta=8.00(\mathrm{~m}, 2 \mathrm{H}), 7.62(\mathrm{~m}, 2 \mathrm{H}), 5.20(\mathrm{q}, \mathrm{J}=7.15,1 \mathrm{H})$, $2.58(\mathrm{~s}, 3 \mathrm{H}) \mathrm{ppm} .{ }^{13} \mathrm{C}$ NMR $\left(500.13 \mathrm{MHz}, \mathrm{CD}_{3} \mathrm{CN}\right) \delta=198.71,141.04,139.88,129.29$ $(2 \mathrm{C}), 128.87(2 \mathrm{C}), 125.80\left(\mathrm{q}, \mathrm{J}_{\mathrm{CF}}=281.8 \mathrm{~Hz}\right), 71.98\left(\mathrm{q}, \mathrm{J}_{\mathrm{CF}}=31.4 \mathrm{~Hz}\right), 27.18 \mathrm{ppm} .{ }^{19} \mathrm{~F}$ NMR (500.13 MHz, $\left.\mathrm{CD}_{3} \mathrm{CN}\right) \delta=-79.11 \mathrm{ppm}$.

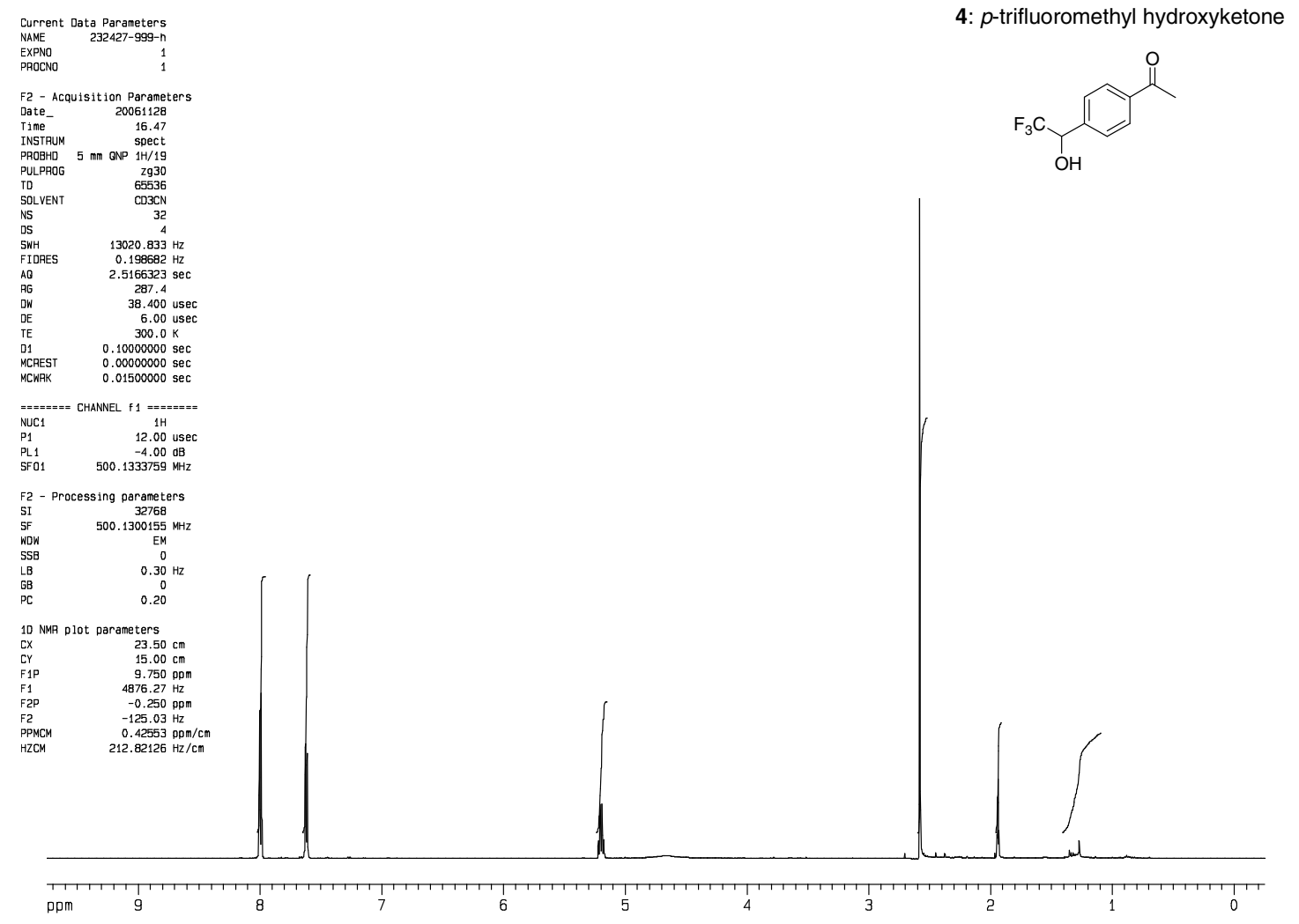




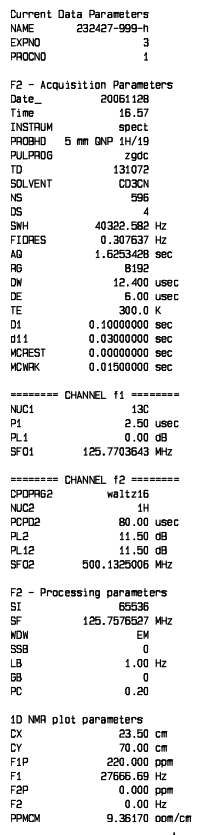

4: $p$-trifluoromethyl hydroxyketone

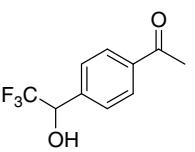

PMDM $9.36170 \mathrm{ocm} / \mathrm{cm}$

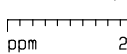

200

180

160

140

120

100

80

60

4: $p$-trifluoromethyl hydroxyketone
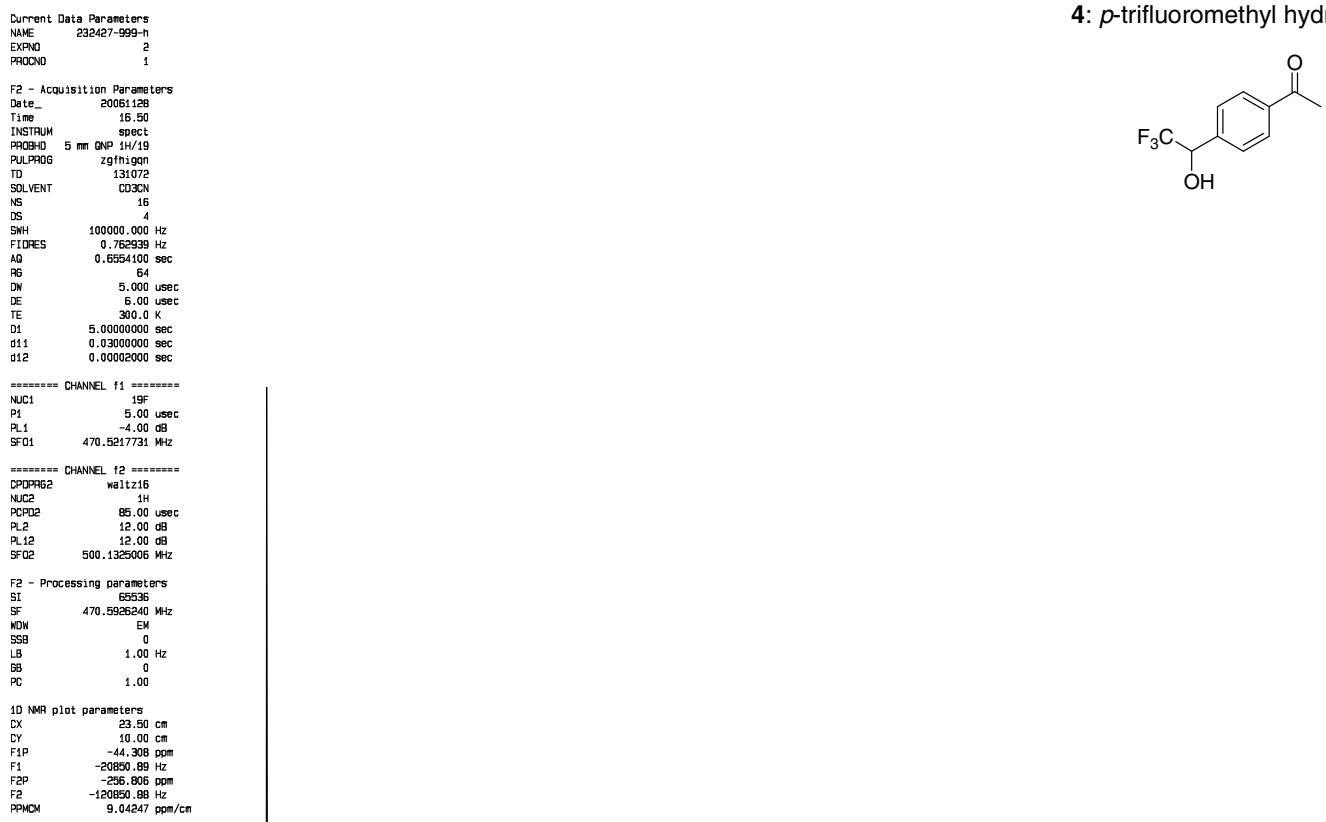

$\mathrm{OH}$

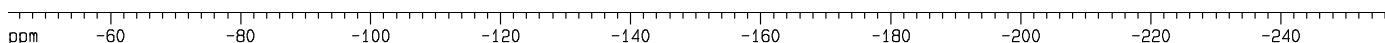


Achiral GC Method:

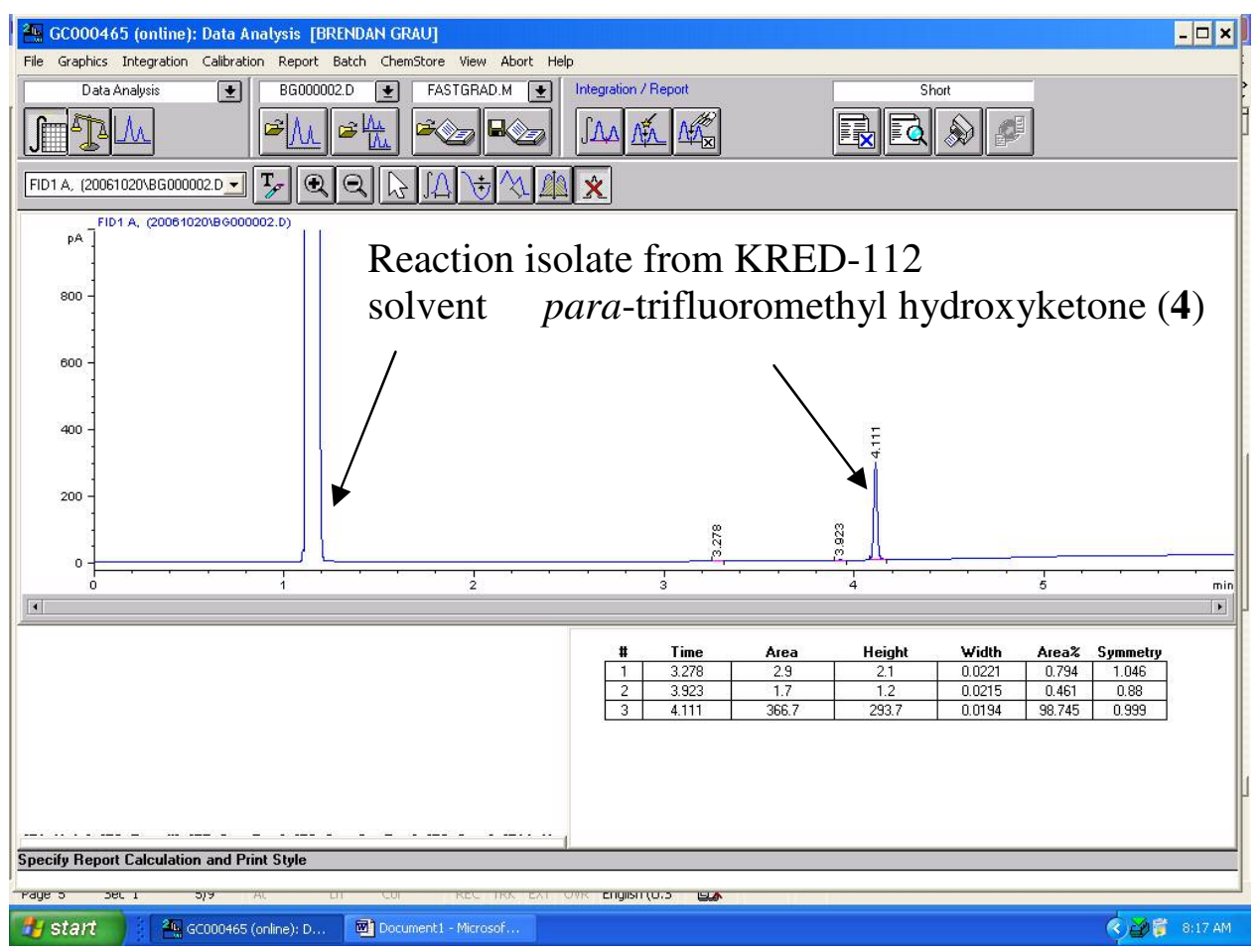

\section{Chiral NP-HPLC Method:}

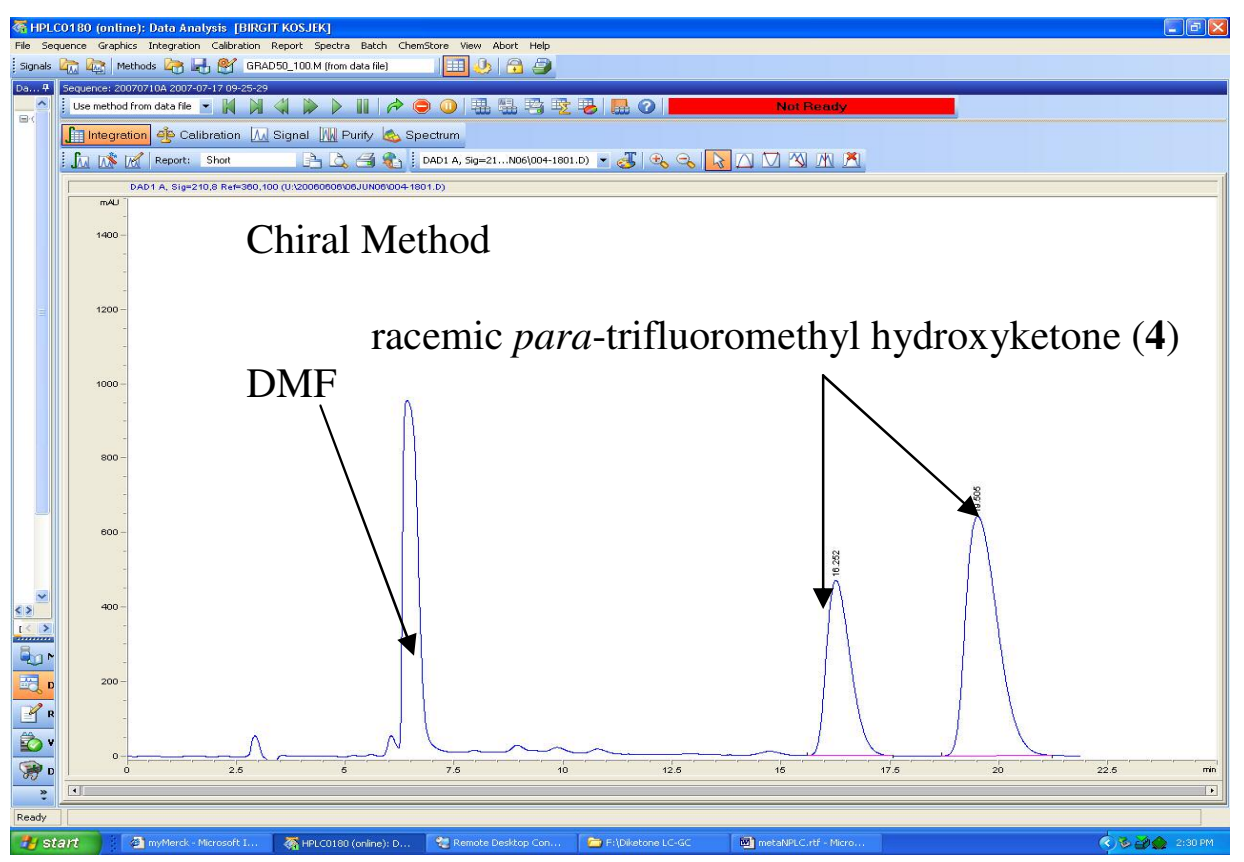


Chiral NP-HPLC Method:

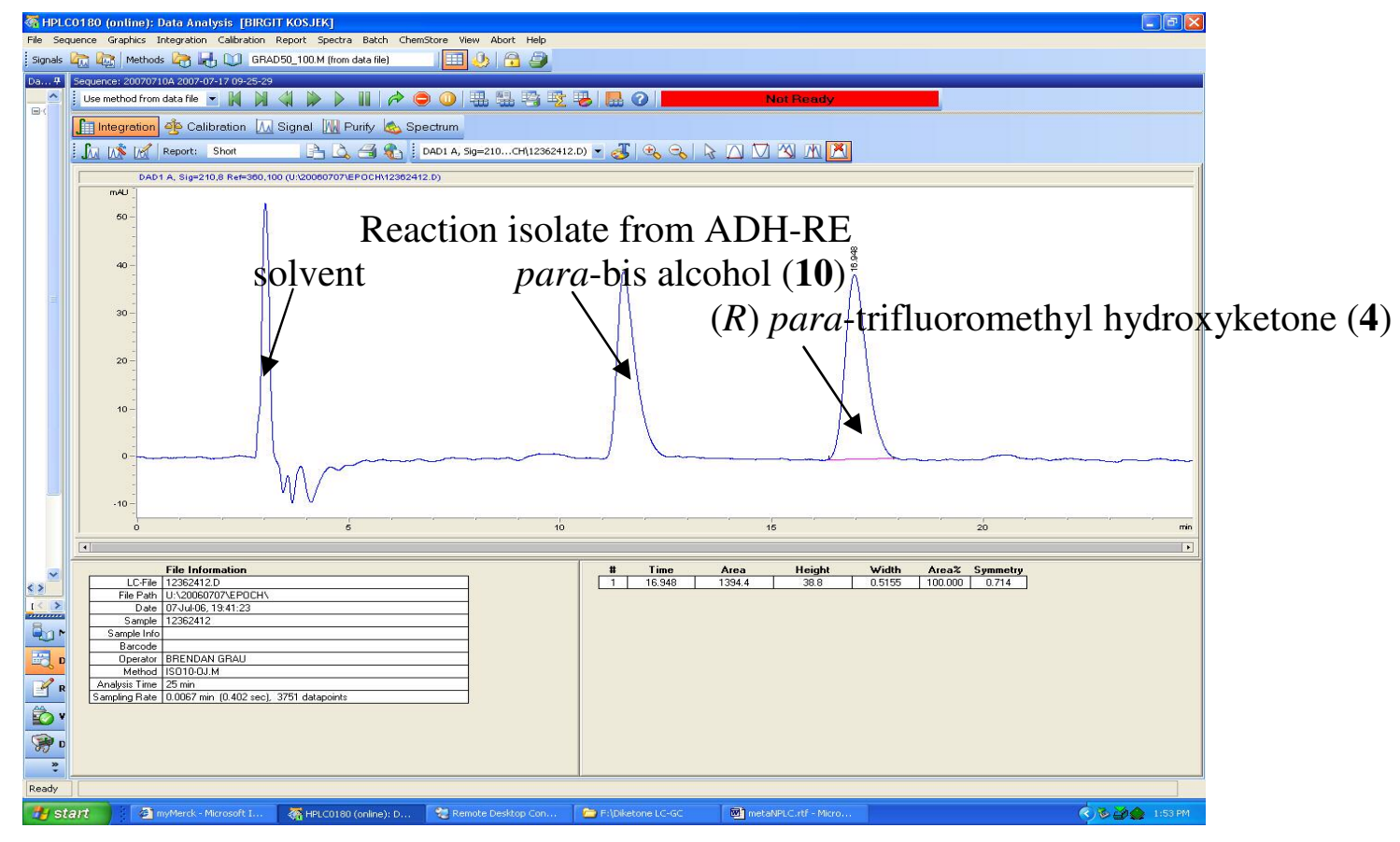


Section 8: meta-trfluoromethyl hydroxyketone 5

5: $m$-trifluoromethyl hydroxyketone<smiles>CC(=O)c1cccc(C(O)C(F)(F)F)c1</smiles>

5: ${ }^{1} \mathrm{H}$ NMR $\left(500.13 \mathrm{MHz}, \mathrm{CD}_{3} \mathrm{CN}\right) \delta=8.06(\mathrm{~s}, 1 \mathrm{H}), 8.00(\mathrm{~d}, \mathrm{~J}=7.55,1 \mathrm{H}), 7.73(\mathrm{~d}, \mathrm{~J}=$ $7.55,1 \mathrm{H}), 7.56(\mathrm{t}, \mathrm{J}=7.55,1 \mathrm{H}), 5.21(\mathrm{~m}, 1 \mathrm{H}), 2.58(\mathrm{~s}, 3 \mathrm{H}) \mathrm{ppm} .{ }^{13} \mathrm{C}$ NMR $(500.13 \mathrm{MHz}$, $\left.\mathrm{CD}_{3} \mathrm{CN}\right) \delta=198.73,138.47,136.98,133.05,130.11,129.93,128.15,125.80\left(\mathrm{q}, \mathrm{J}_{\mathrm{CF}}=\right.$ $281.8 \mathrm{~Hz}), 72.00\left(\mathrm{q}, \mathrm{J}_{\mathrm{CF}}=31.4 \mathrm{~Hz}\right), 27.15 \mathrm{ppm} .{ }^{19} \mathrm{~F} \mathrm{NMR}\left(500.13 \mathrm{MHz}, \mathrm{CD}_{3} \mathrm{CN}\right) \delta=-$ $79.34 \mathrm{ppm}$.

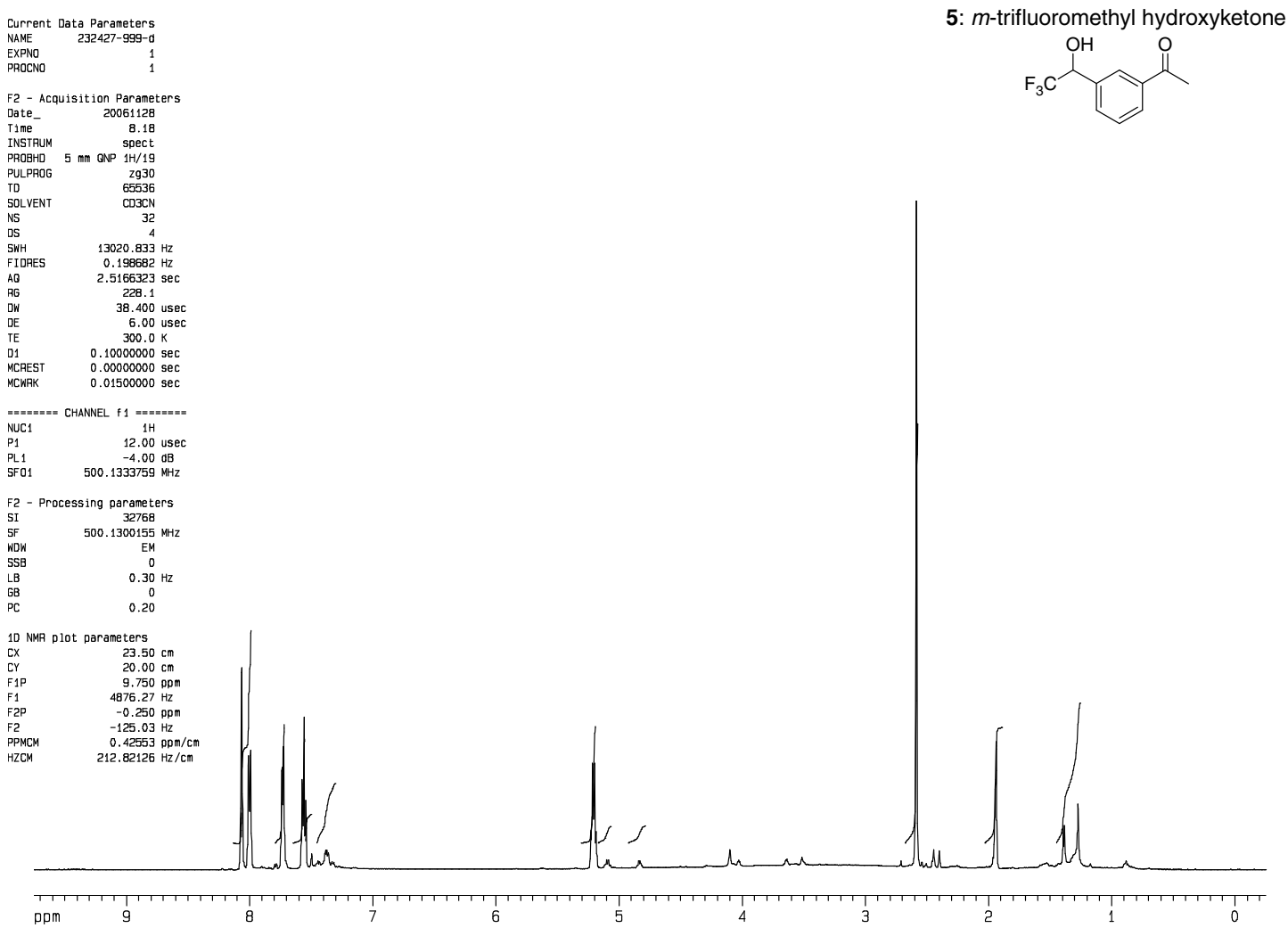




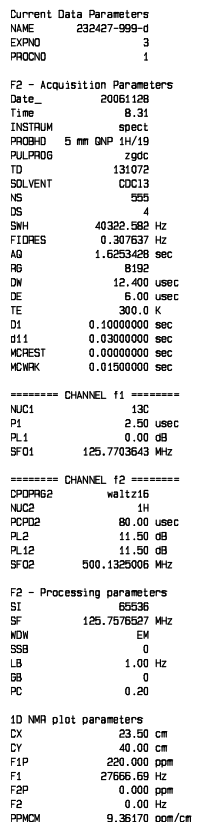

5: m-trifluoromethyl hydroxyketone

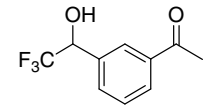

$9.36170 \mathrm{omom} / \mathrm{cm}$
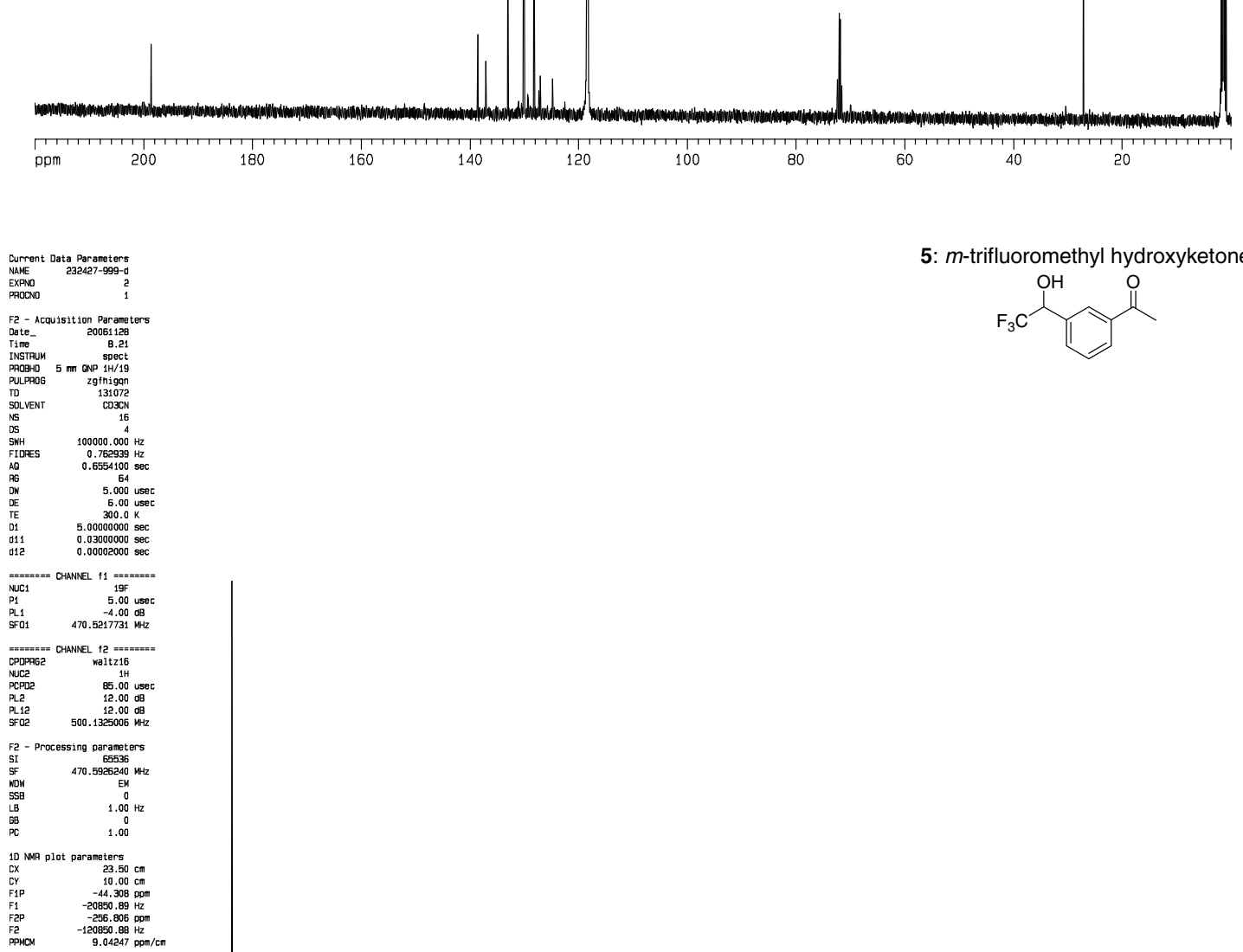

5: $m$-trifluoromethyl hydroxyketone
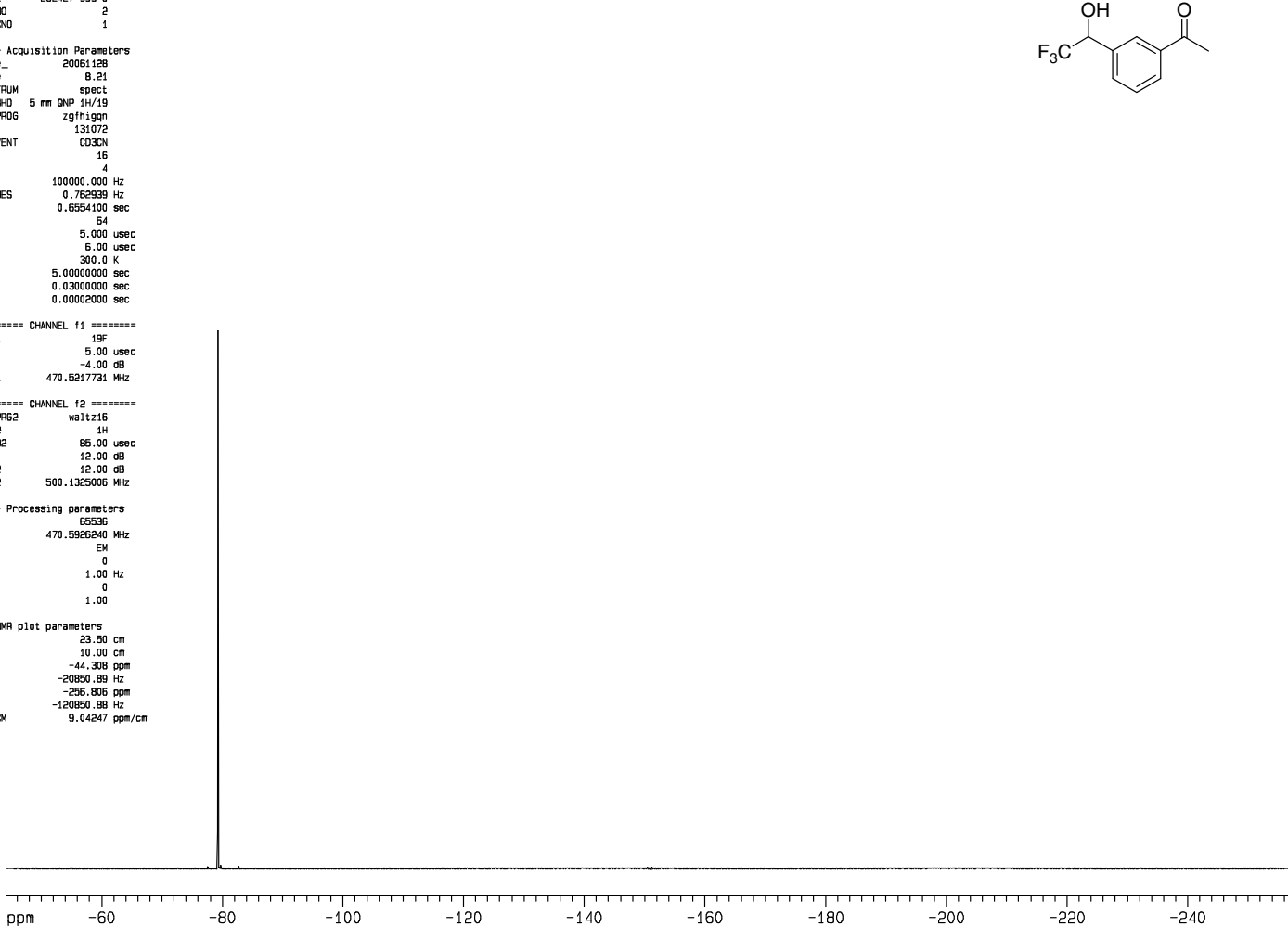
Achiral GC Method:

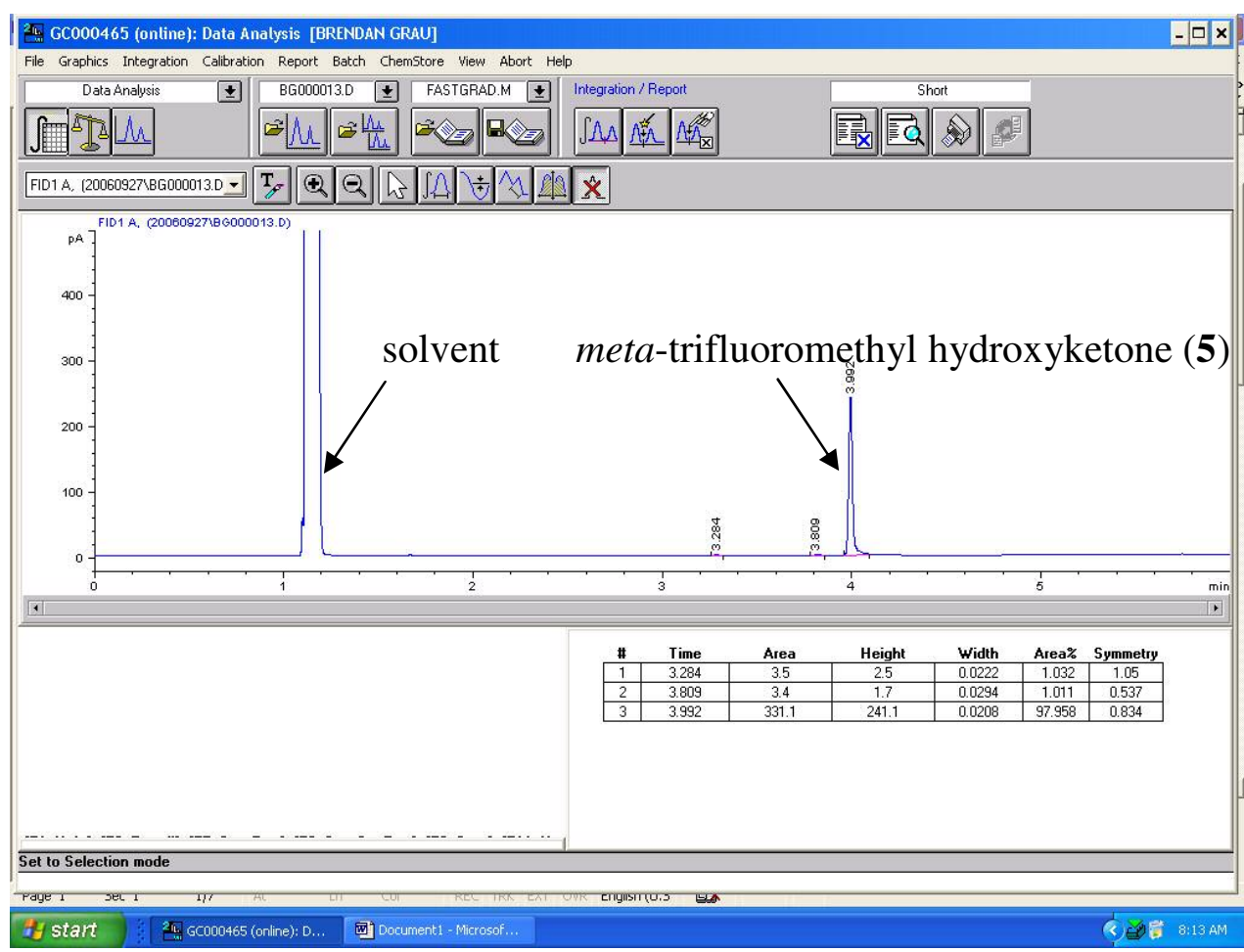

Chiral NP-HPLC Method:

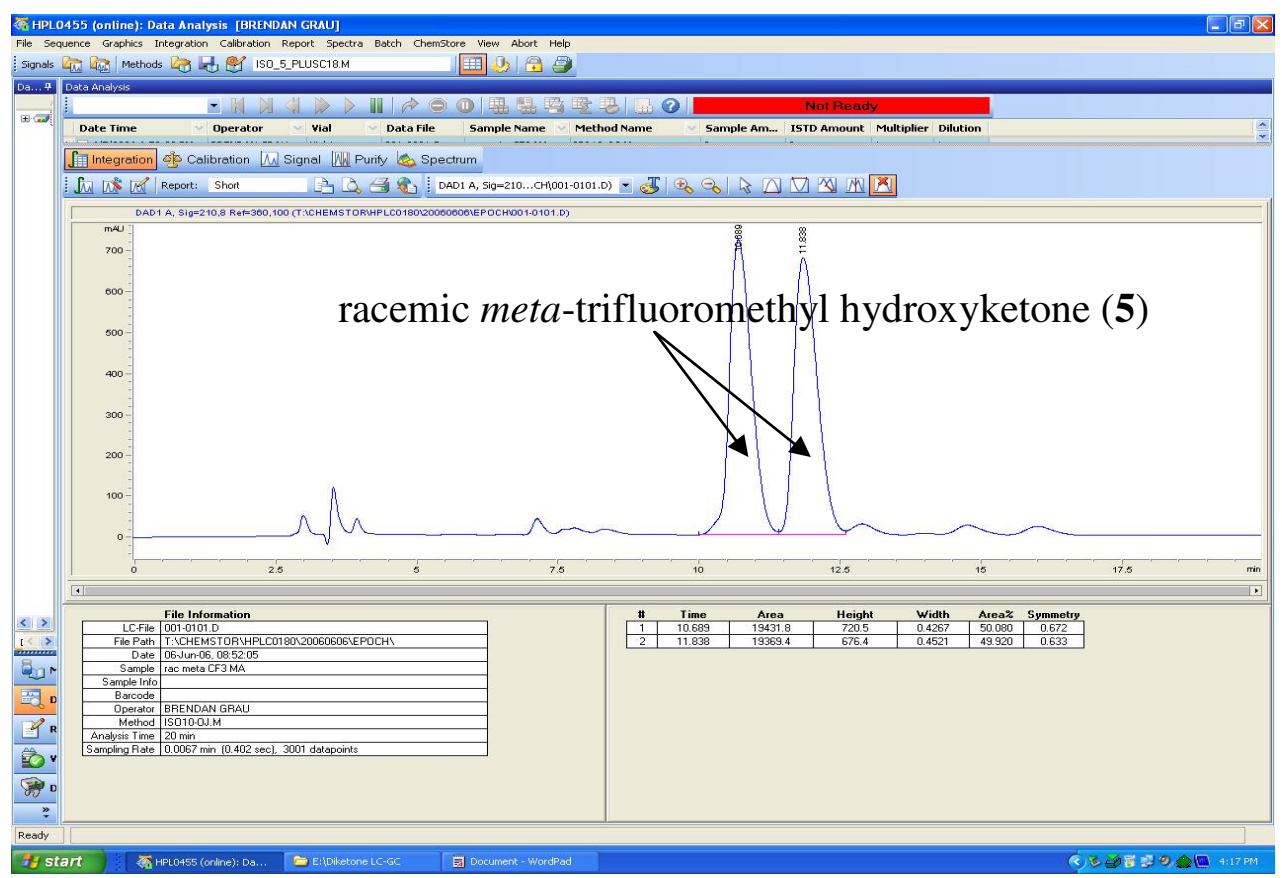


Chiral NP-HPLC Method:

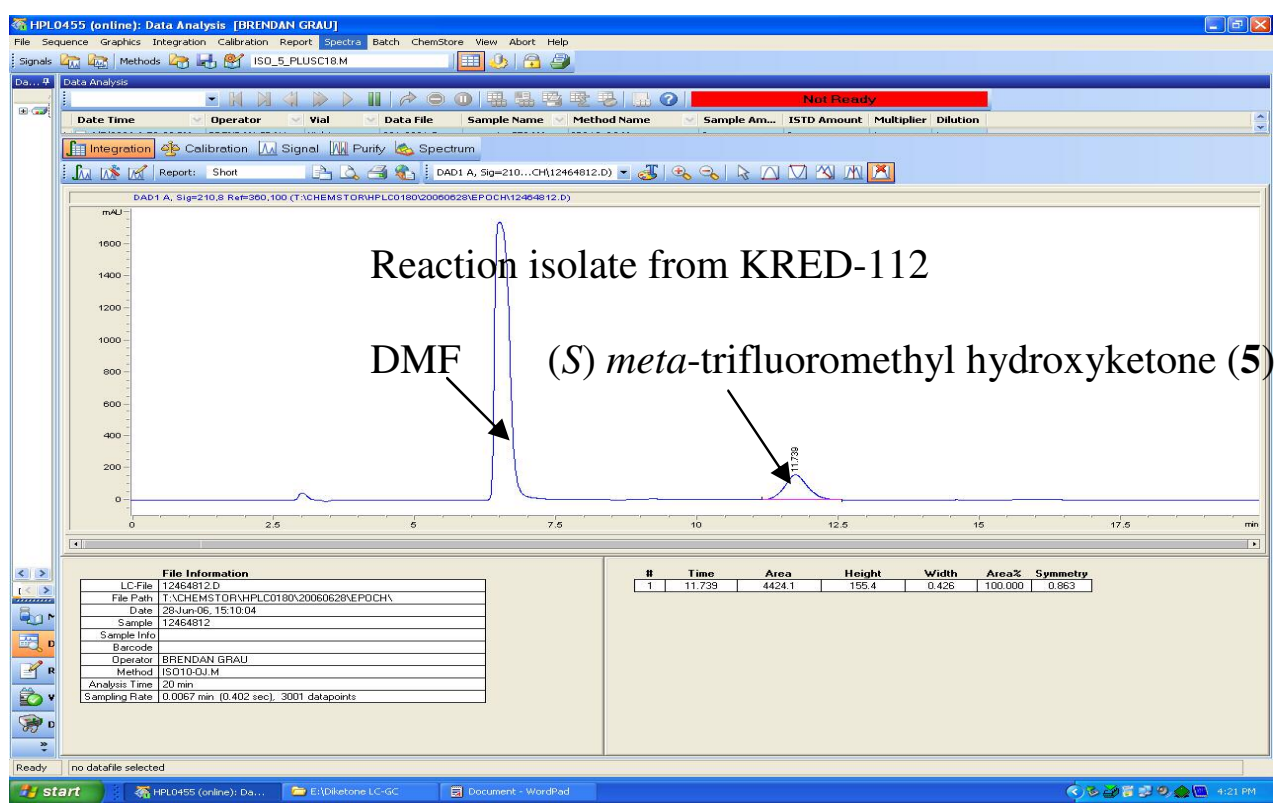


Section 9: Synthesis of racemic methyl hydroxyketones $\mathbf{7}$ and $\mathbf{8}$.

The racemic standards for the methyl hydroxyketones $\mathbf{7}$ and $\mathbf{8}$ were subjected to modified reaction conditions listed in the literature reference ${ }^{2}$ by using $\mathrm{THF} / \mathrm{H}_{2} \mathrm{O}$ as solvent. No reaction was observed on compound 3a. The THF solution was extracted with MTBE ( 2 vol.) and the organic layer was washed with brine ( $1 / 2 \mathrm{vol}$.). The organic layer was dried over $\mathrm{MgSO}_{4}$ and concentrated on a rotary evaporator to yield the crude racemic methyl hydroxyketones of $\mathbf{7}$ and $\mathbf{8}$. 
Section 10: para-methyl hydroxyketone 7

7: $p$-methyl hydroxyketone<smiles>CC(O)c1ccc(C(=O)C(F)(F)F)cc1</smiles><smiles>CC(O)c1ccc(C(O)(O)C(F)(F)F)cc1</smiles>

7: ketone form

7: hydrate form

(hydrate form): ${ }^{1} \mathrm{H}$ NMR $\left(500.13 \mathrm{MHz}, \mathrm{CD}_{3} \mathrm{CN}\right) \delta=7.61(\mathrm{~m}, 2 \mathrm{H}), 7.41(\mathrm{~m}, 2 \mathrm{H}), 5.25(\mathrm{~s}$, $2 \mathrm{H}), 4.84(\mathrm{~m}, 1 \mathrm{H}), 3.31(\mathrm{brs}, 1 \mathrm{H}), 1.39(\mathrm{~d}, \mathrm{~J}=6.36,3 \mathrm{H}) \mathrm{ppm} .{ }^{13} \mathrm{C} \mathrm{NMR}(500.13 \mathrm{MHz}$, $\left.\mathrm{CD}_{3} \mathrm{CN}\right) \delta=149.49,136.90,128.22(2 \mathrm{C}), 126.06(2 \mathrm{C}), 124.40\left(\mathrm{q}, \mathrm{J}_{\mathrm{CF}}=287.3 \mathrm{~Hz}\right), 94.24$ $\left(\mathrm{q}, \mathrm{J}_{\mathrm{CF}}=32.0 \mathrm{~Hz}\right), 69.86,25.93 \mathrm{ppm} .{ }^{19} \mathrm{~F}$ NMR $\left(500.13 \mathrm{MHz}, \mathrm{CD}_{3} \mathrm{CN}\right) \delta=-85.39 \mathrm{ppm}$.

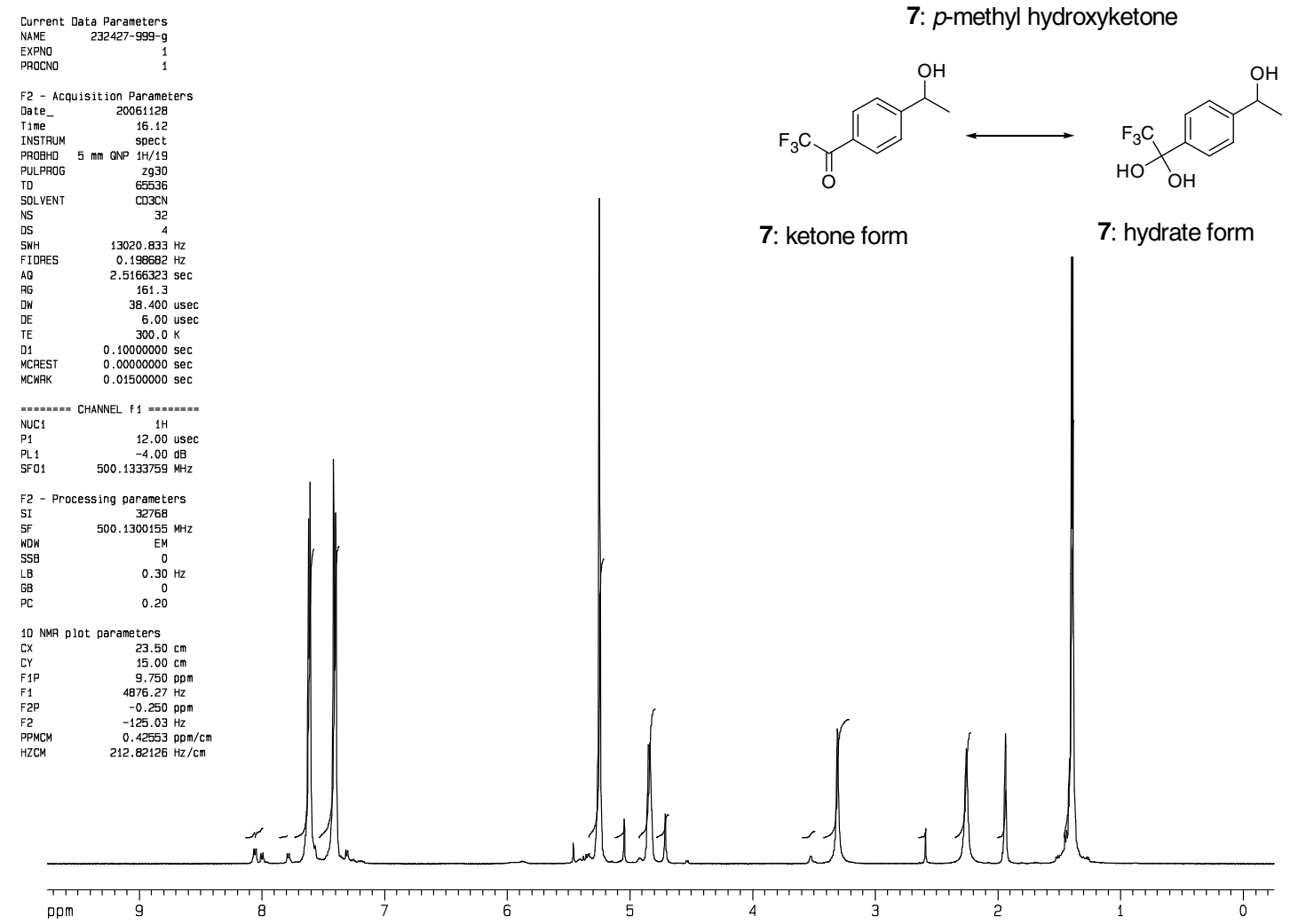




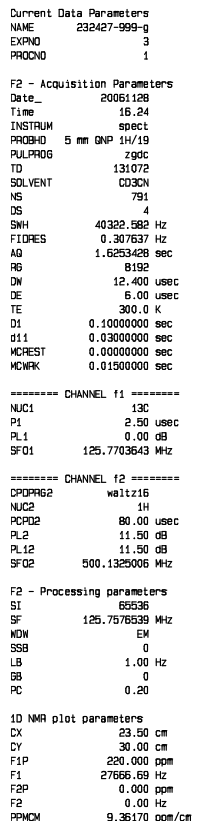

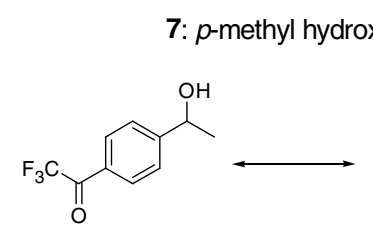

7: ketone form

7: hydrate form

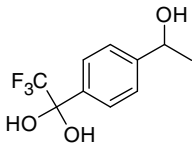

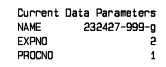

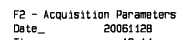

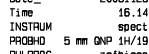

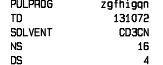

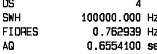

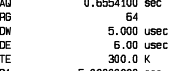

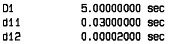

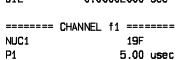

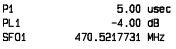

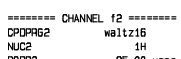

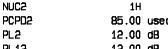

SF02 500.1325005 MH+

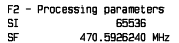

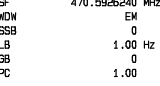

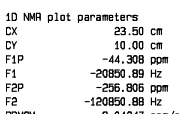

7: p-methyl hydroxyketone<smiles>CC(O)c1ccc(C(=O)C(F)(F)F)cc1</smiles>

7: ketone form

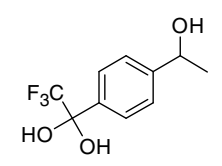

7: hydrate form 
Achiral GC Method:

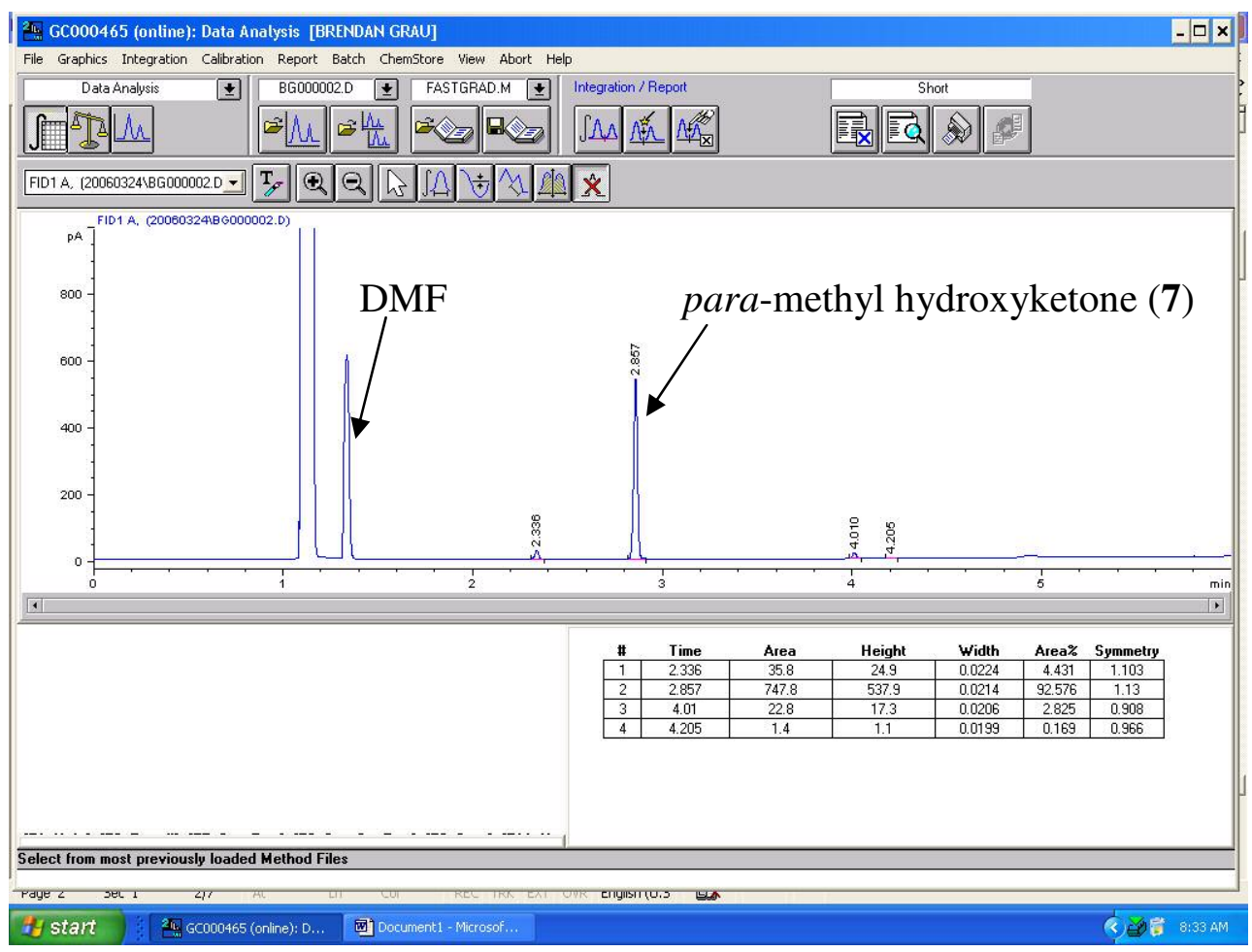

Chiral GC Method:

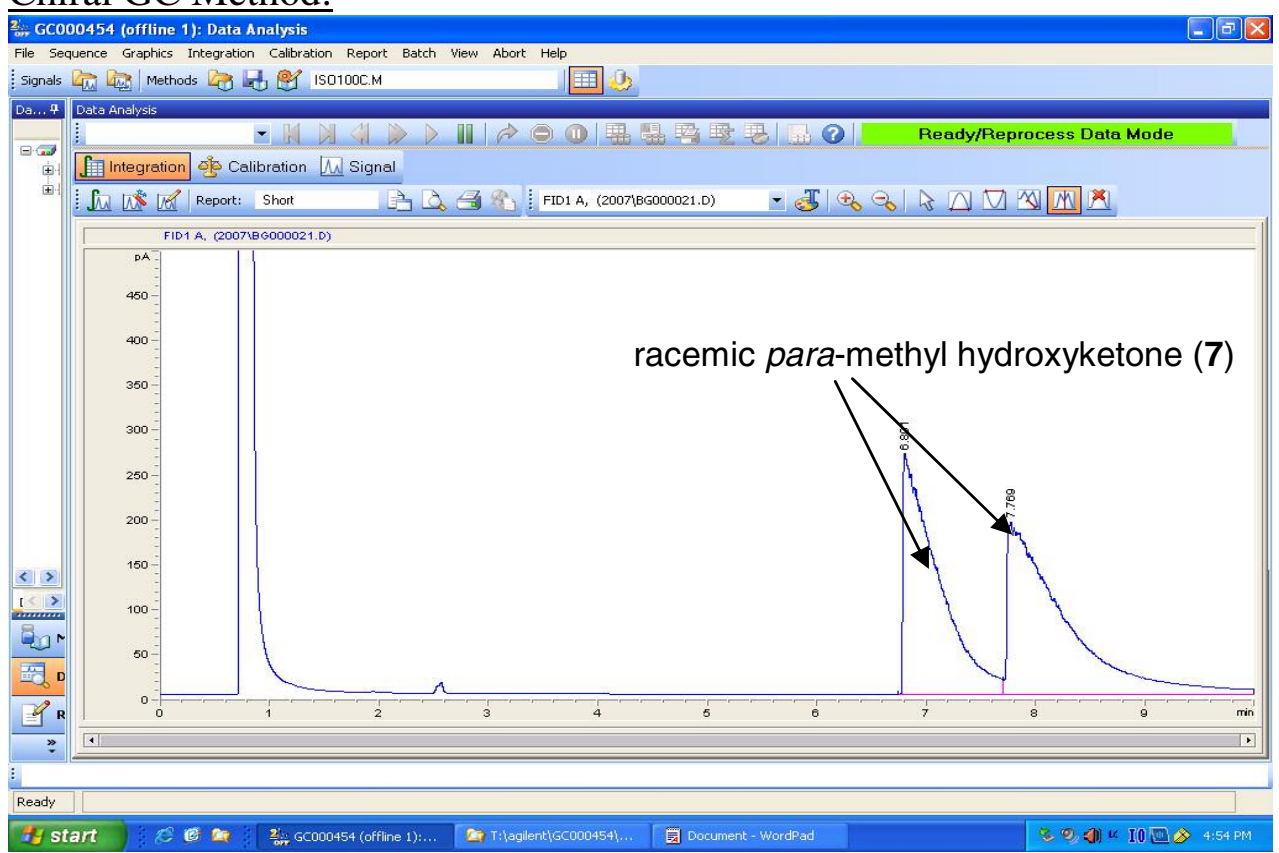


Chiral GC Method:

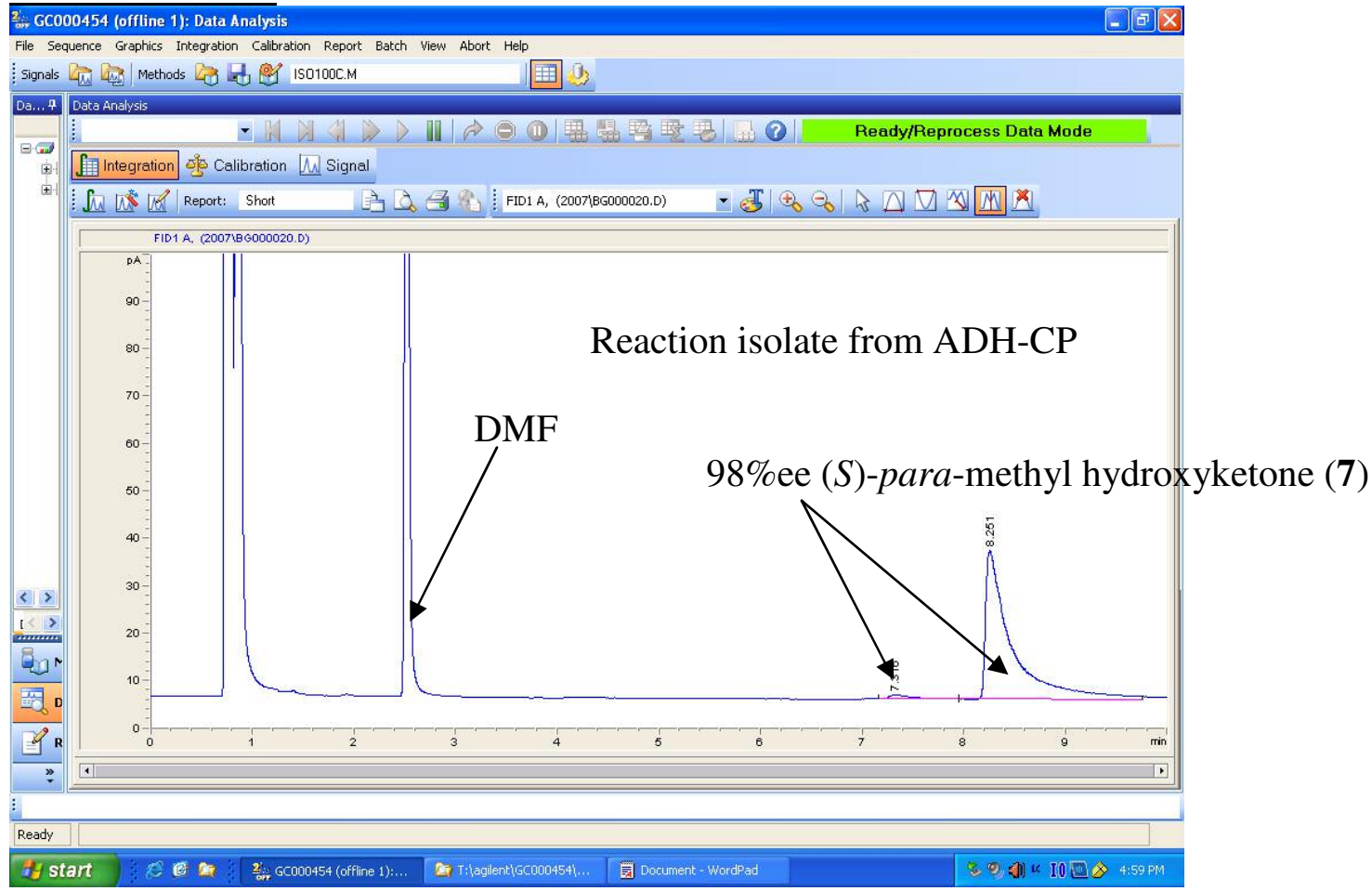


Section 11: meta-methyl hydroxyketone 8

8: $m$-methyl hydroxyketone<smiles>CC(O)c1cccc(C(C)(C)C(F)(F)F)c1</smiles>

8: ketone form

8: hydrate form

(hydrate form): ${ }^{1} \mathrm{H}$ NMR $\left(500.13 \mathrm{MHz}, \mathrm{CD}_{3} \mathrm{CN}\right) \delta=7.66(\mathrm{~s}, 1 \mathrm{H}), 7.53(\mathrm{~d}, \mathrm{~J}=7.55,1 \mathrm{H})$, $7.41(\mathrm{~m}, 1 \mathrm{H}), 7.39(\mathrm{~d}, \mathrm{~J}=7.55,1 \mathrm{H}), 4.85(\mathrm{q}, \mathrm{J}=6.36,1 \mathrm{H}), 3.64(\mathrm{~m}, 2 \mathrm{H}), 1.39(\mathrm{~m}, 3 \mathrm{H})$ ppm. ${ }^{13} \mathrm{C}$ NMR $\left(500.13 \mathrm{MHz}, \mathrm{CD}_{3} \mathrm{CN}\right) \delta=147.92,138.33,130.39,128.99,127.84$, $126.84,125.32,94.50$ (q, J $\mathrm{CF}=32.4 \mathrm{~Hz}), 69.5,26.0 \mathrm{ppm} .{ }^{19} \mathrm{~F} \mathrm{NMR}(500.13 \mathrm{MHz}$, $\left.\mathrm{CD}_{3} \mathrm{CN}\right) \delta=-85.36 \mathrm{ppm}$.

(ketone form): ${ }^{1} \mathrm{H}$ NMR $\left(500.13 \mathrm{MHz}, \mathrm{CD}_{3} \mathrm{CN}\right) \delta=8.08(\mathrm{~s}, 1 \mathrm{H}), 7.96(\mathrm{~d}, \mathrm{~J}=7.55,1 \mathrm{H})$, $7.78(\mathrm{~d}, \mathrm{~J}=7.55,1 \mathrm{H}), 7.59(\mathrm{~m}, 1 \mathrm{H}), 4.92(\mathrm{q}, \mathrm{J}=6.36,1 \mathrm{H}), 3.64(\mathrm{~m}, 2 \mathrm{H}), 1.39(\mathrm{~m}, 3 \mathrm{H})$ ppm. ${ }^{19} \mathrm{~F}$ NMR $\left(500.13 \mathrm{MHz}, \mathrm{CD}_{3} \mathrm{CN}\right) \delta=-72.70 \mathrm{ppm}$.

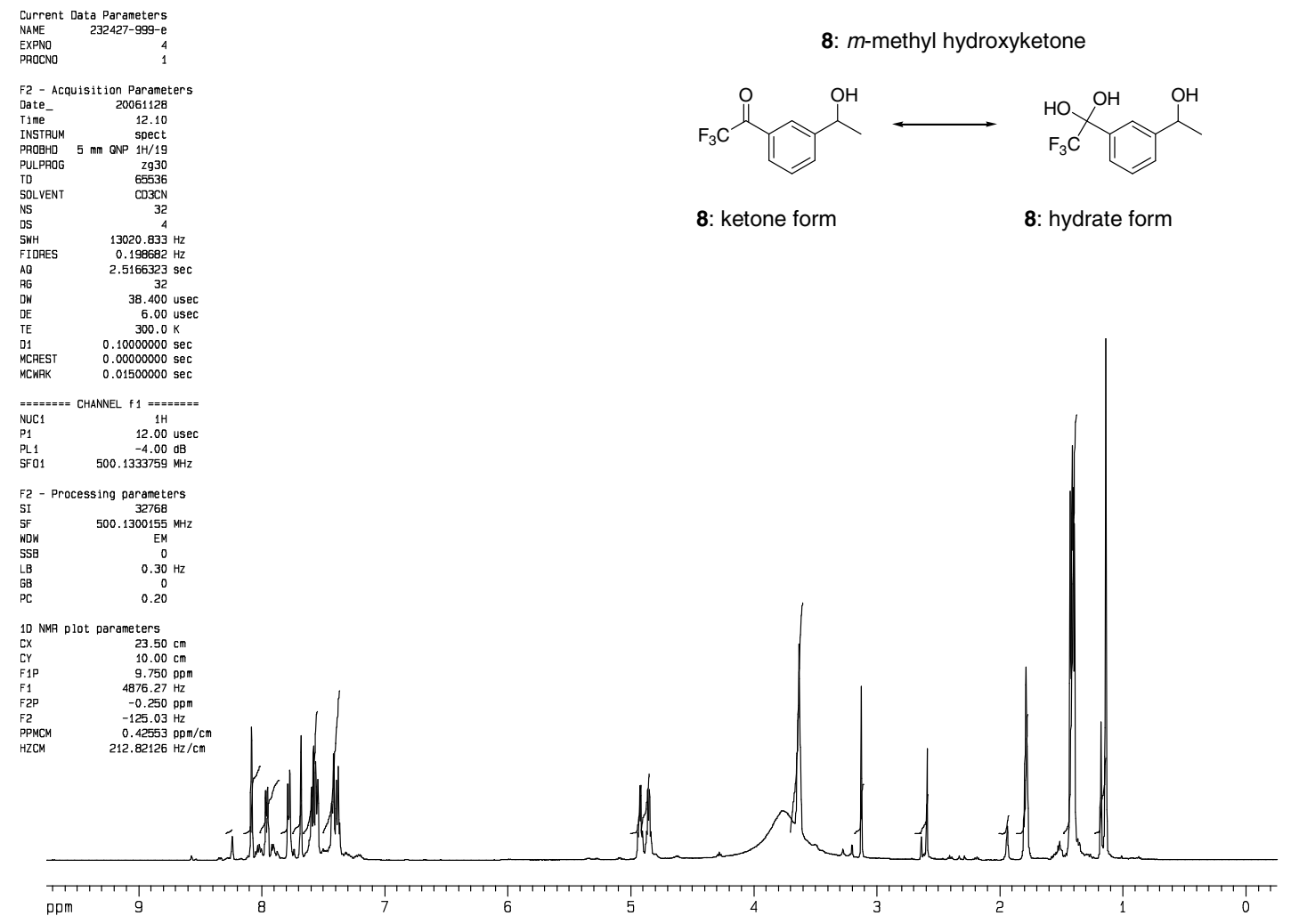



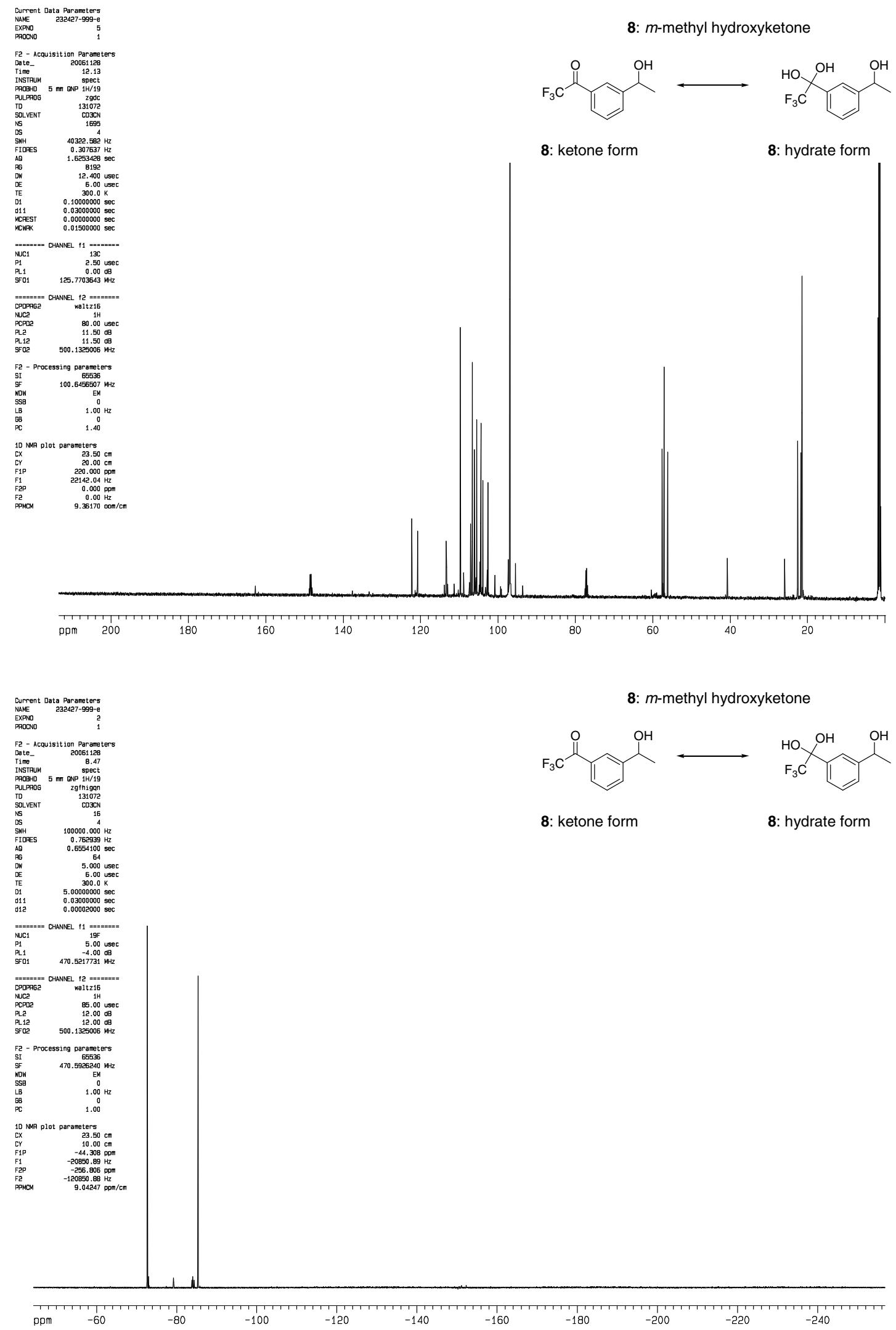
Achiral GC Method:

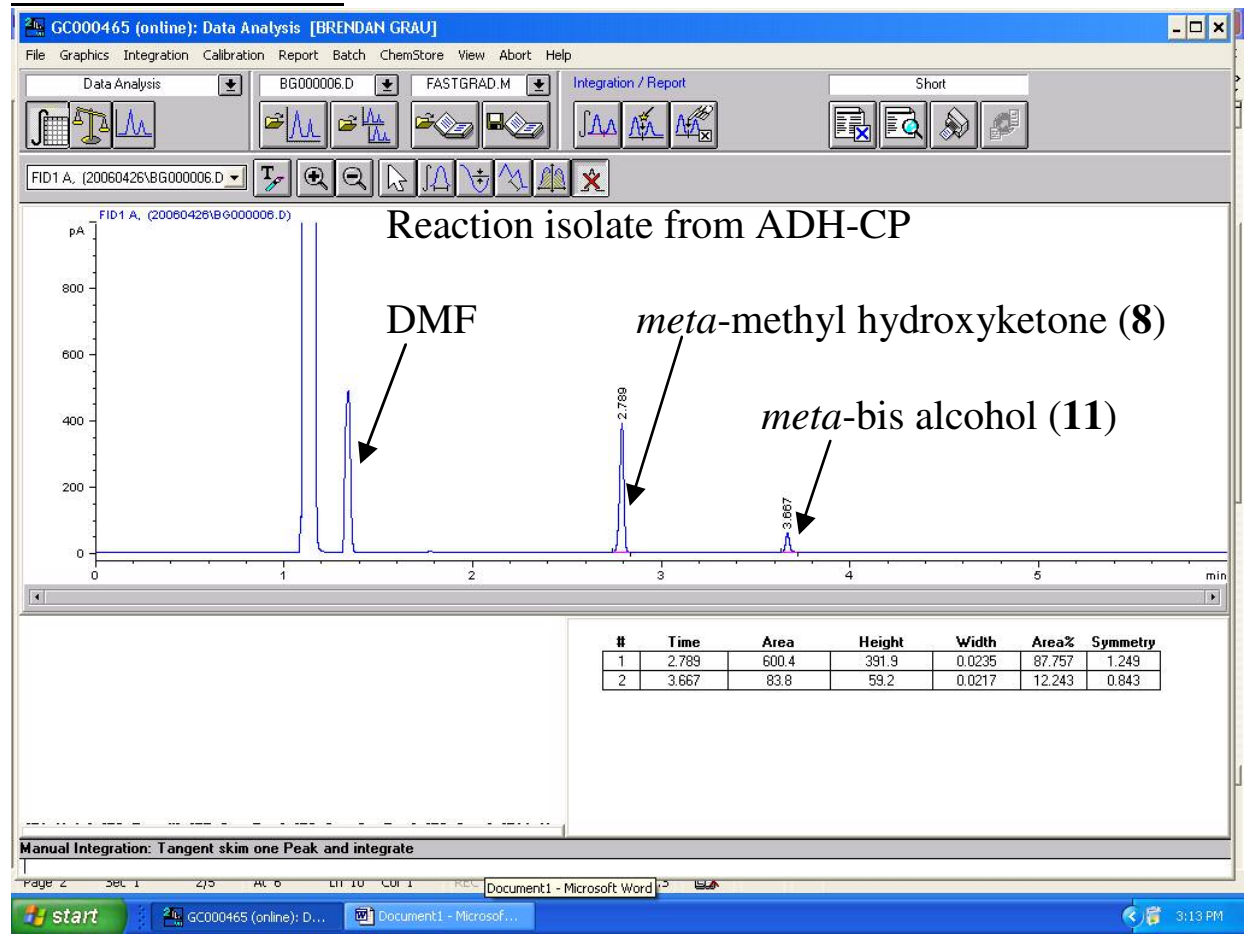

Chiral GC Method:

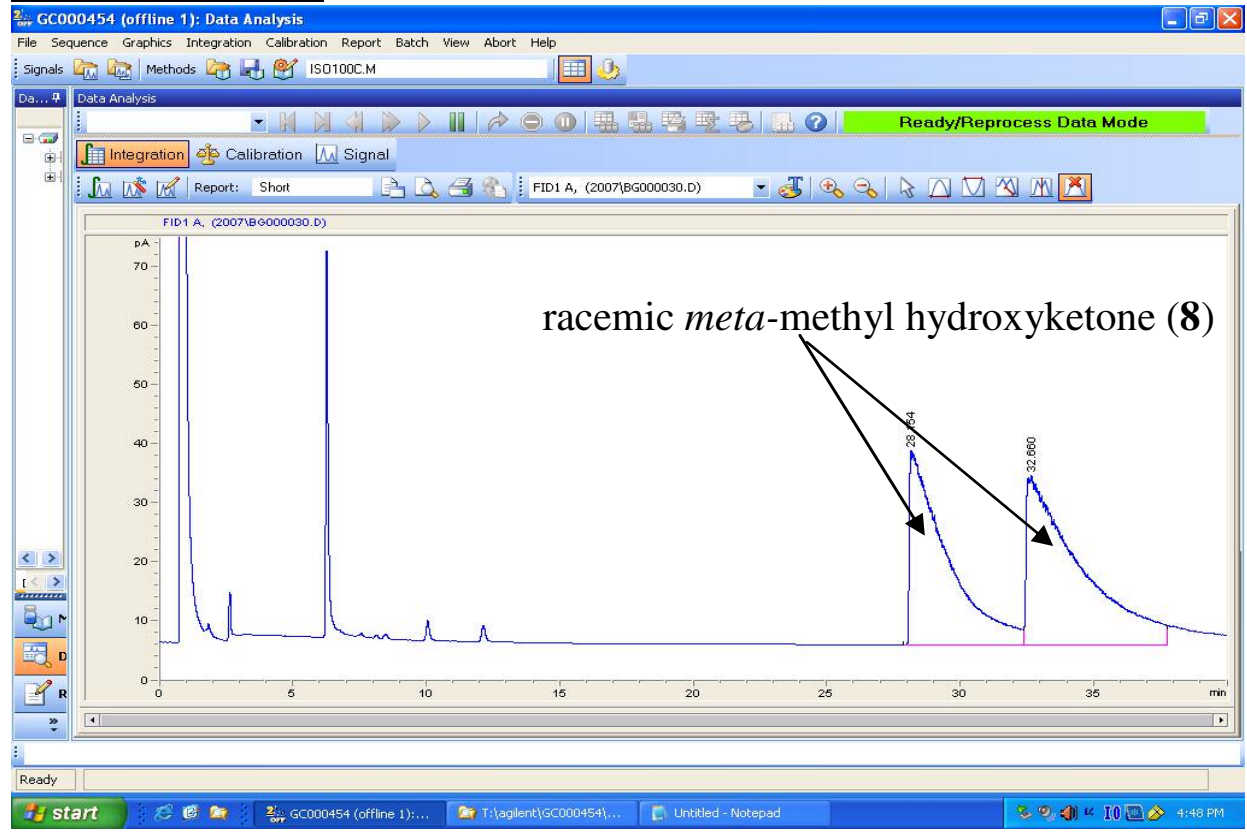


Chiral GC Method:

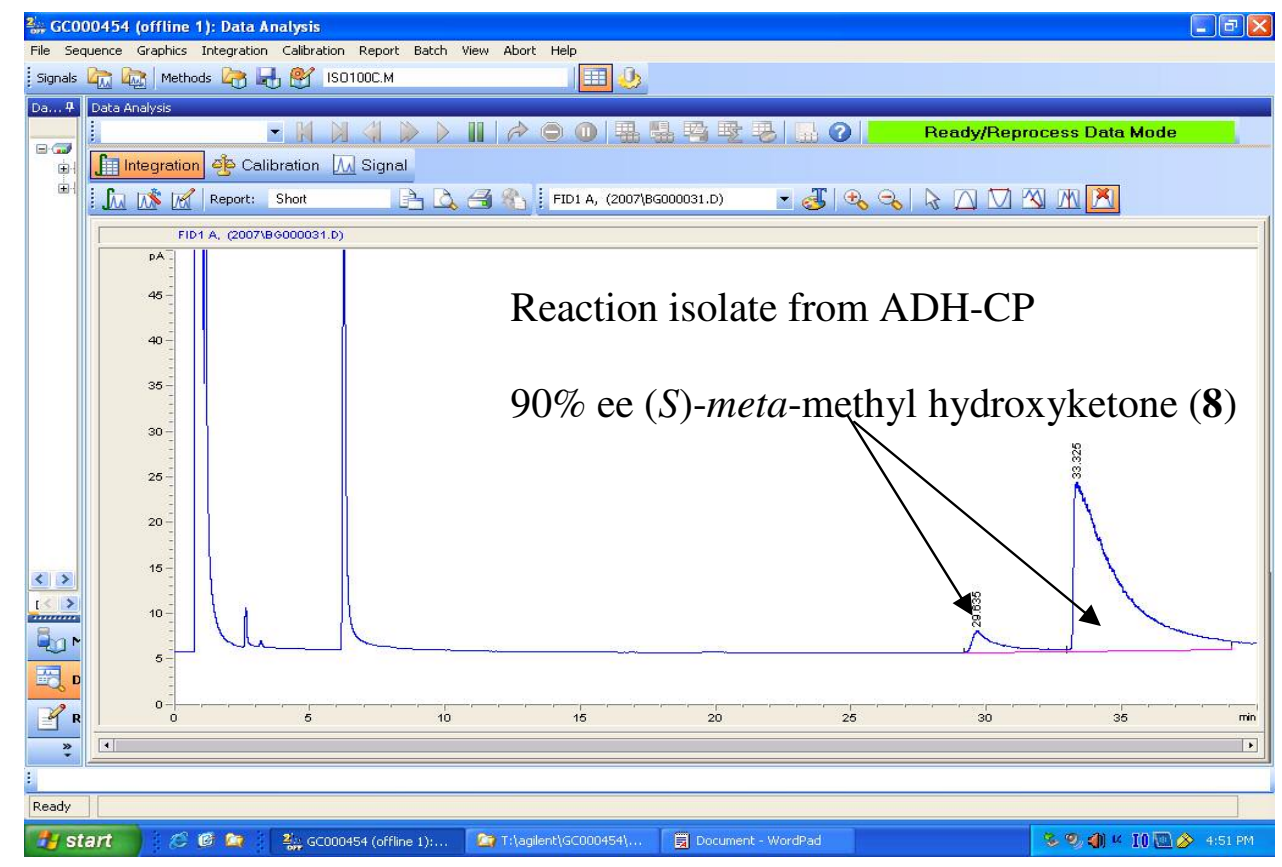


Section 12: Synthesis of bis alcohol $\mathbf{1 0}$ and $\mathbf{1 1 .}$

The bis alcohol standards for $\mathbf{1 0}$ and $\mathbf{1 1}$ were made by reacting the diketones with excess sodium borohydride. No reaction was observed on compound $\mathbf{3 a}$.

Section 13: para bis alcohol 10

10: $p$-bis-alcohol<smiles>CC(O)c1ccc(C(O)C(F)(F)F)cc1</smiles>

10: ${ }^{1} \mathrm{H}$ NMR $\left(500.13 \mathrm{MHz}, \mathrm{CD}_{3} \mathrm{CN}\right) \delta=7.45(\mathrm{~m}, 2 \mathrm{H}), 7.40(\mathrm{~m}, 2 \mathrm{H}), 5.08(\mathrm{~m}, 1 \mathrm{H}), 4.83$ $(\mathrm{m}, 1 \mathrm{H}), 4.52$ (brs, $1 \mathrm{H}), 3.26$ (brs, $1 \mathrm{H}), 1.39$ (d, J = 6.36, 3H) ppm. ${ }^{13} \mathrm{C}$ NMR $(500.13$ $\left.\mathrm{MHz}, \mathrm{CD}_{3} \mathrm{CN}\right) \delta=148.04$ [148.04], 133.79 [133.79], 127.45 (2C) [127.45 (2C)], 125.37 $(2 \mathrm{C})[125.37(2 \mathrm{C})], 125.00\left(\mathrm{q}, \mathrm{J}_{\mathrm{CF}}=281.2 \mathrm{~Hz}\right)\left[125.00\left(\mathrm{q}, \mathrm{J}_{\mathrm{CF}}=281.2 \mathrm{~Hz}\right)\right], 71.22\left(\mathrm{q}, \mathrm{J}_{\mathrm{CF}}\right.$ $=31.4 \mathrm{~Hz})\left[71.20\left(\mathrm{q}, \mathrm{J}_{\mathrm{CF}}=30.8 \mathrm{~Hz}\right)\right], 68.81$ [68.78], 24.90 [24.90] ppm. ${ }^{19} \mathrm{~F} \mathrm{NMR}$ $\left(500.13 \mathrm{MHz}, \mathrm{CD}_{3} \mathrm{CN}\right) \delta=-79.28 \mathrm{ppm}$.

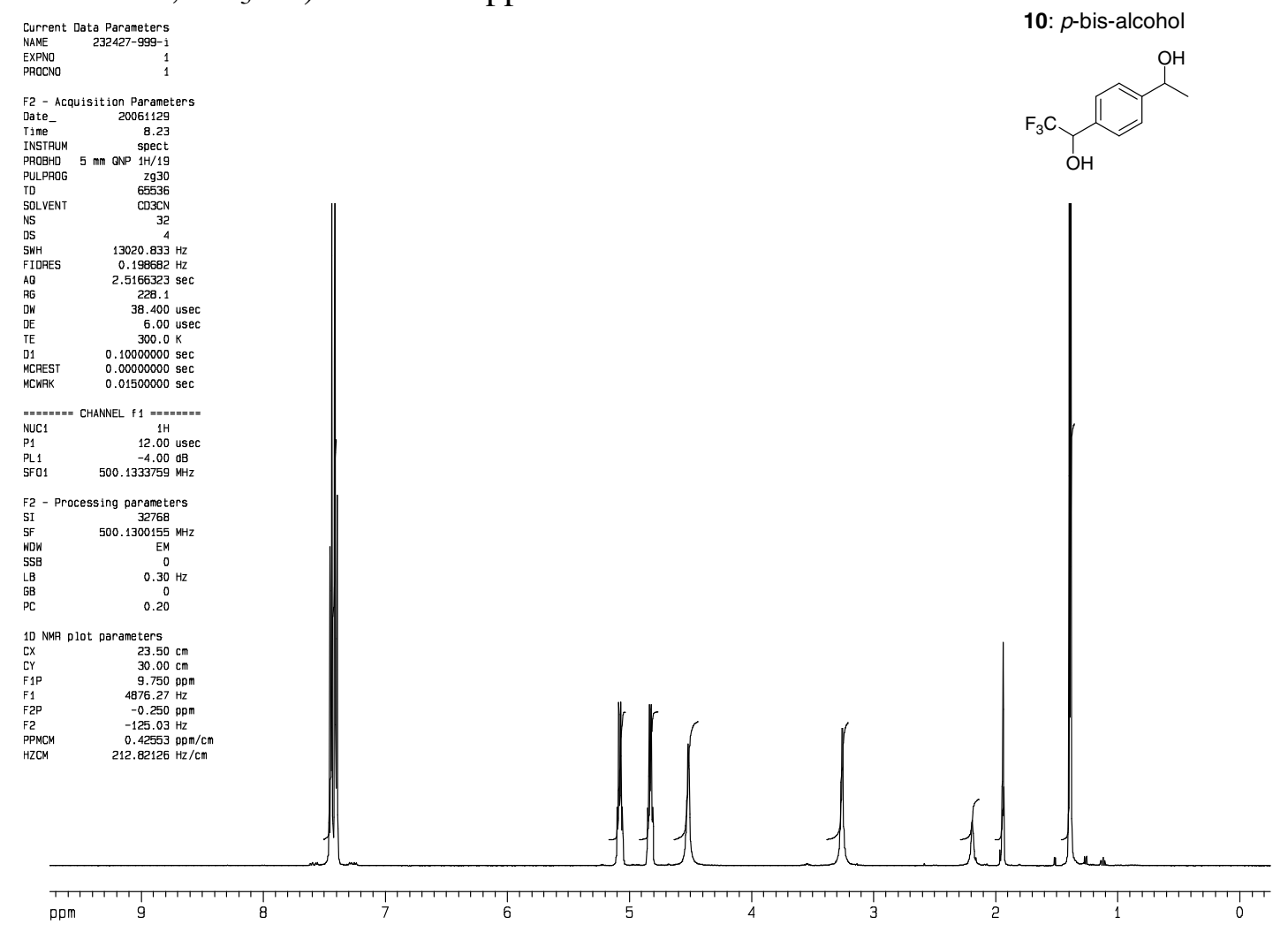



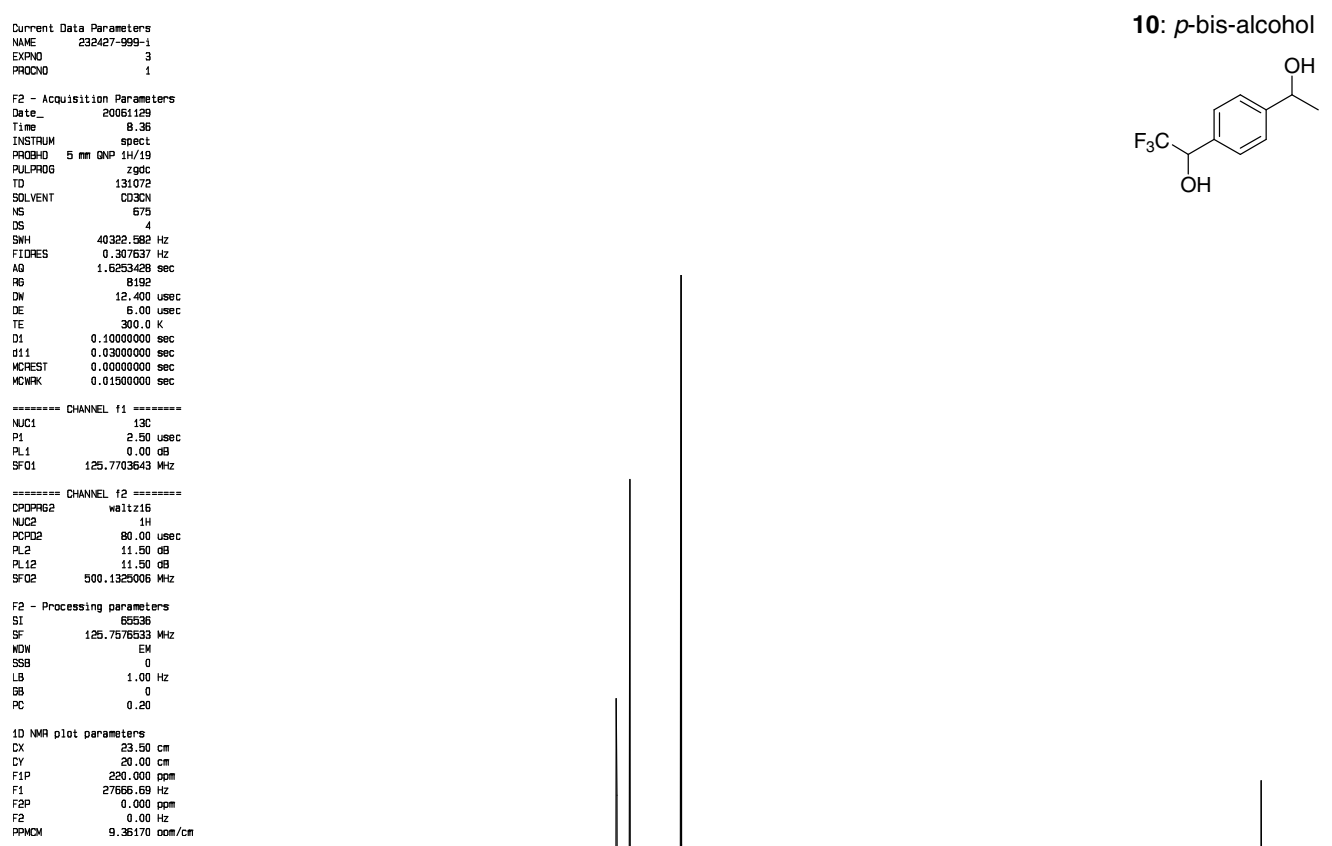

$9.36170 \mathrm{oban} / \mathrm{cm}$

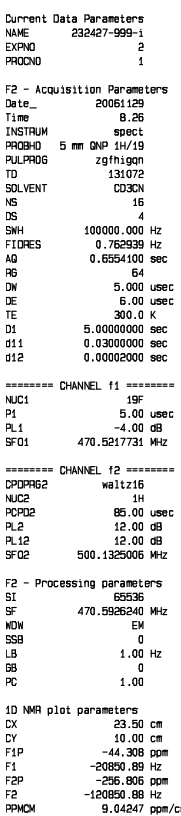

10: $p$-bis-alcohol
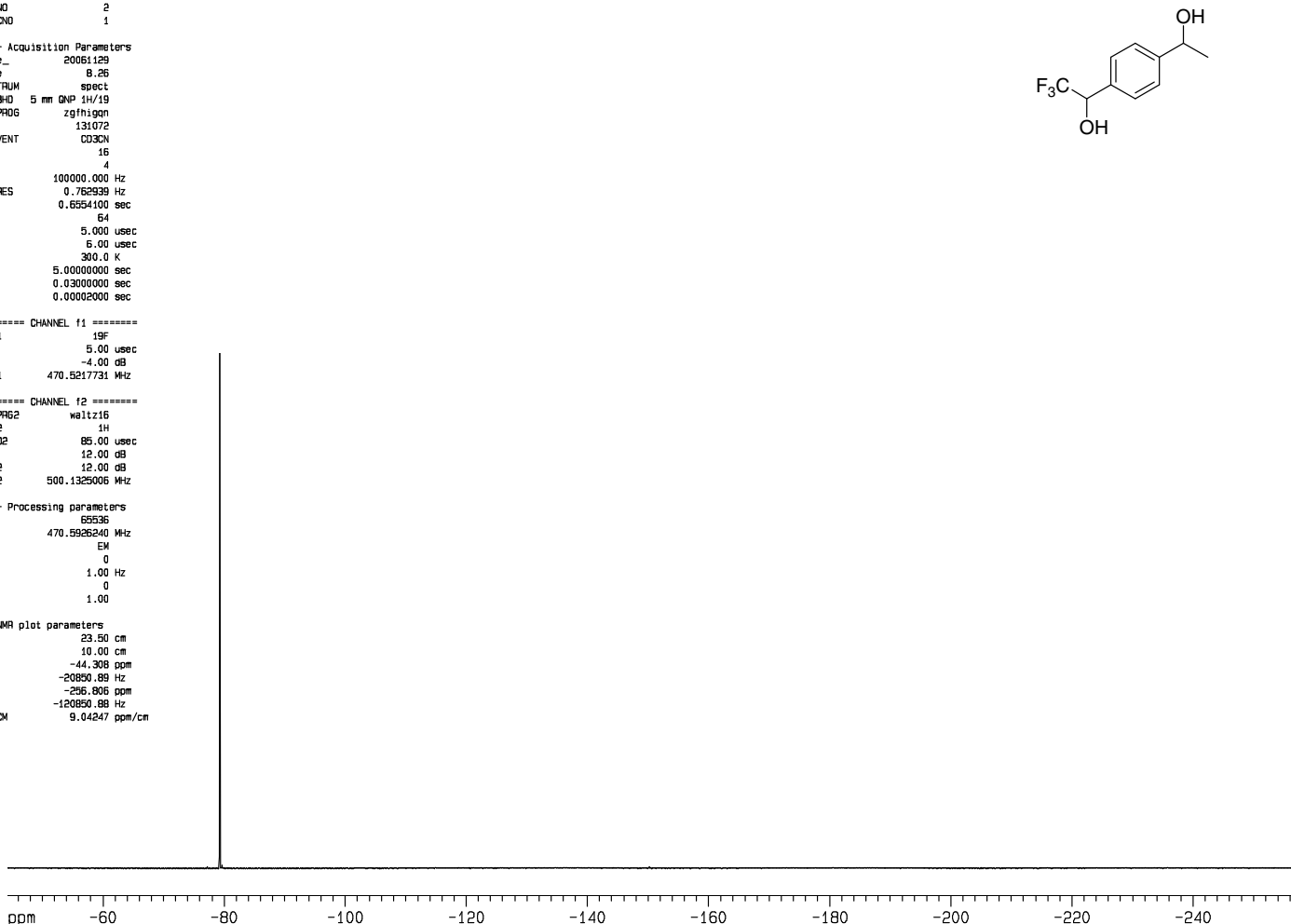
Achiral GC Method:

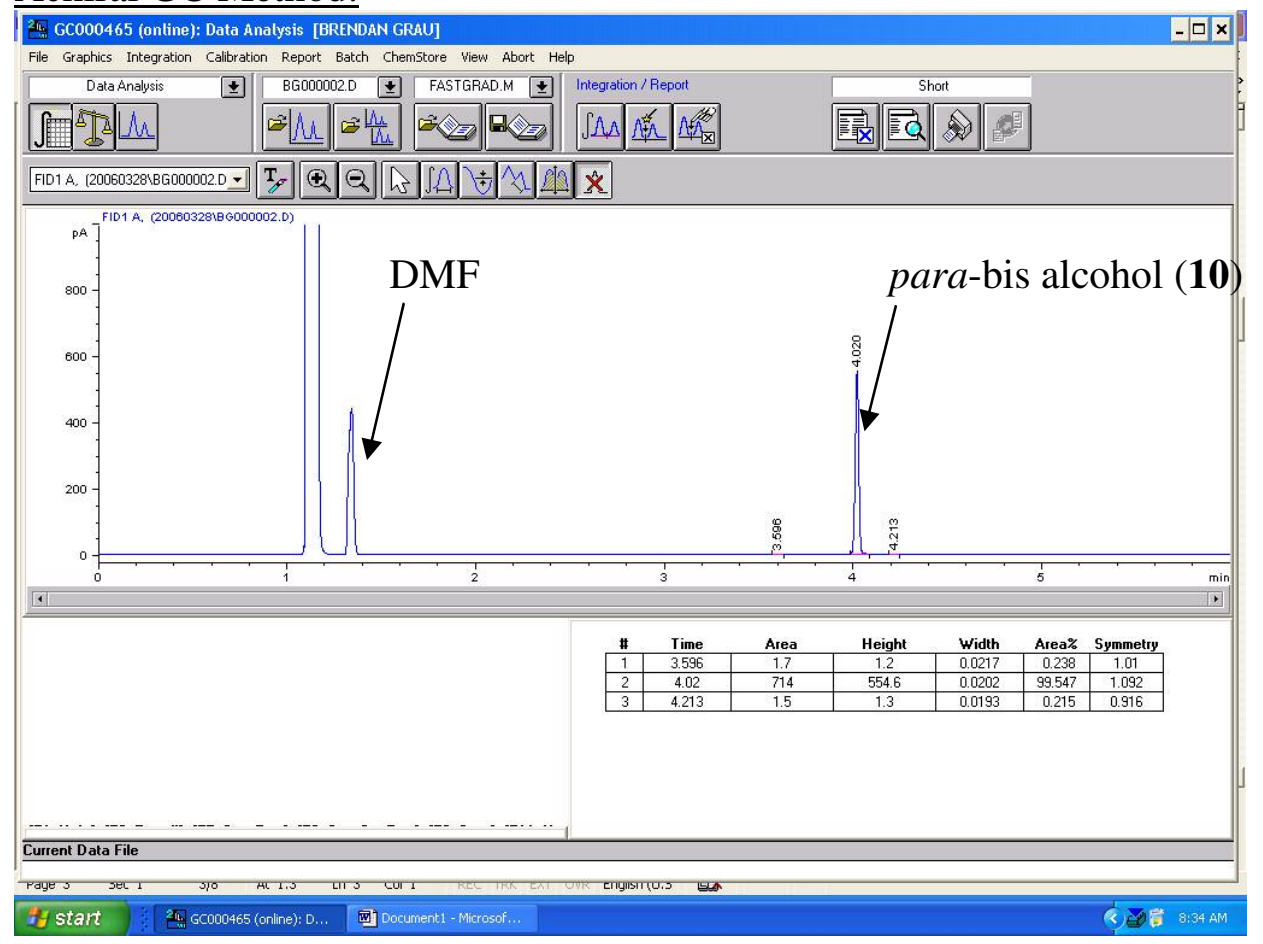


Section 14: meta bis-alcohol 11

11: $m$-bis-alcohol<smiles>CC(O)c1cccc(C(O)C(F)(F)F)c1</smiles>

11: ${ }^{1} \mathrm{H}$ NMR $\left(500.13 \mathrm{MHz}, \mathrm{CD}_{3} \mathrm{CN}\right) \delta=7.50(\mathrm{~s}, 1 \mathrm{H}), 7.38(\mathrm{~m}, 3 \mathrm{H}), 5.10(\mathrm{~m}, 1 \mathrm{H}), 4.84$ $(\mathrm{m}, 1 \mathrm{H}), 4.64(\mathrm{~d}, \mathrm{~J}=5.56,1 \mathrm{H}), 3.37(\mathrm{~d}, \mathrm{~J}=3.58,1 \mathrm{H}), 1.39(\mathrm{~d}, \mathrm{~J}=6.36,3 \mathrm{H}) \mathrm{ppm} .{ }^{13} \mathrm{C}$ NMR (500.13 MHz, CD $\left.{ }_{3} \mathrm{CN}\right) \delta=148.28$ [148.28], 136.30 [136.30], 129.37 (2C) [129.37 $(2 \mathrm{C})$ ], 127.26 [127.19], 125.67 [125.63], $126.08\left(\mathrm{q}, \mathrm{J}_{\mathrm{CF}}=281.8 \mathrm{~Hz}\right)\left[126.08\left(\mathrm{q}, \mathrm{J}_{\mathrm{CF}}=\right.\right.$ $281.8 \mathrm{~Hz})$ ], 70.03 [70.00], $72.52\left(\mathrm{q}, \mathrm{J}_{\mathrm{CF}}=30.8 \mathrm{~Hz}\right)\left[72.52\left(\mathrm{q}, \mathrm{J}_{\mathrm{CF}}=30.8 \mathrm{~Hz}\right)\right], 26.04$ [26.02] ppm. ${ }^{19} \mathrm{~F}$ NMR (500.13 MHz, $\left.\mathrm{CD}_{3} \mathrm{CN}\right) \delta=-79.20 \mathrm{ppm}$.

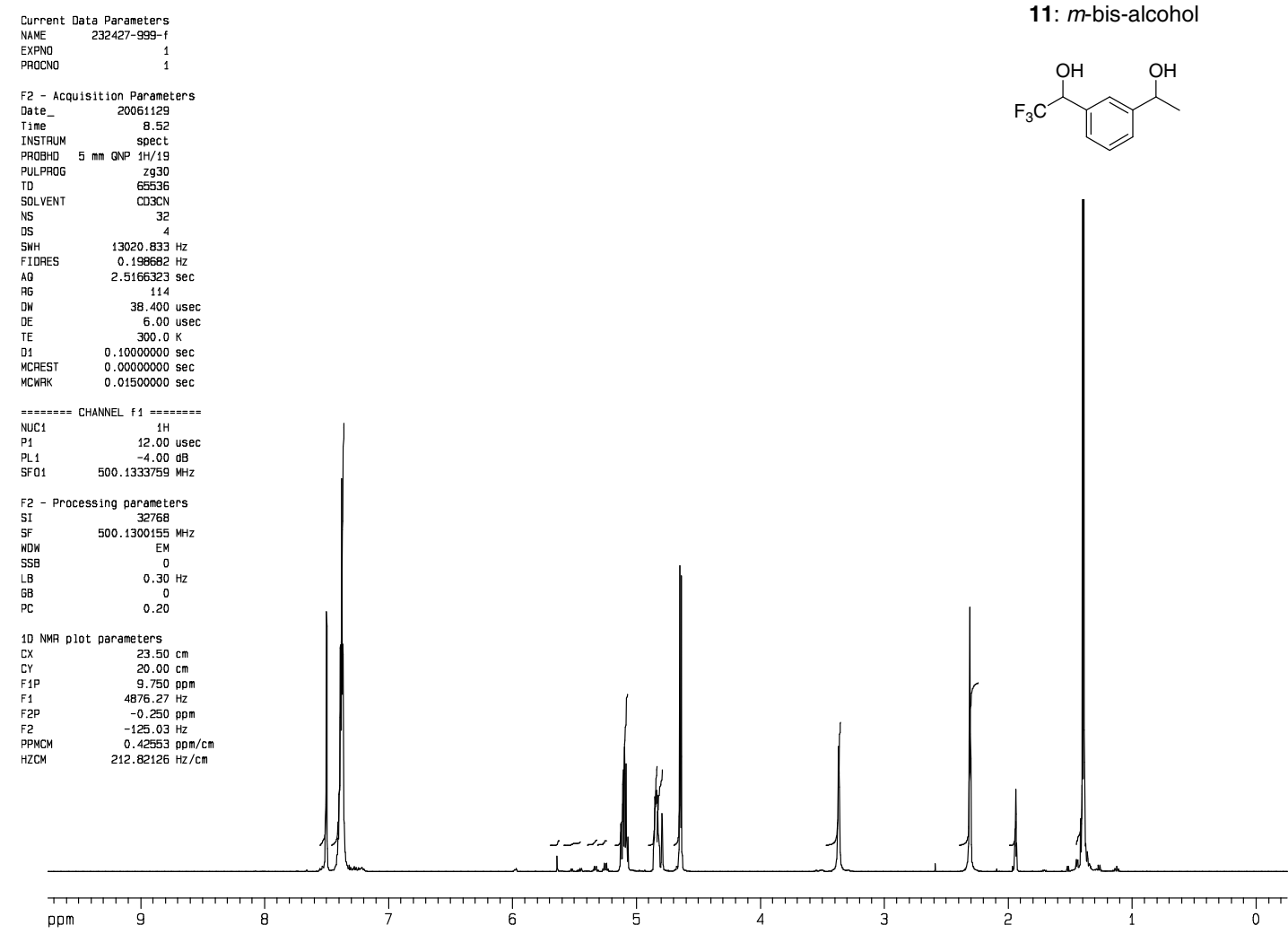




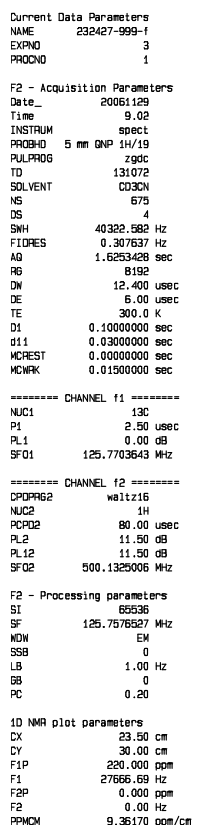

11: $m$-bis-alcohol
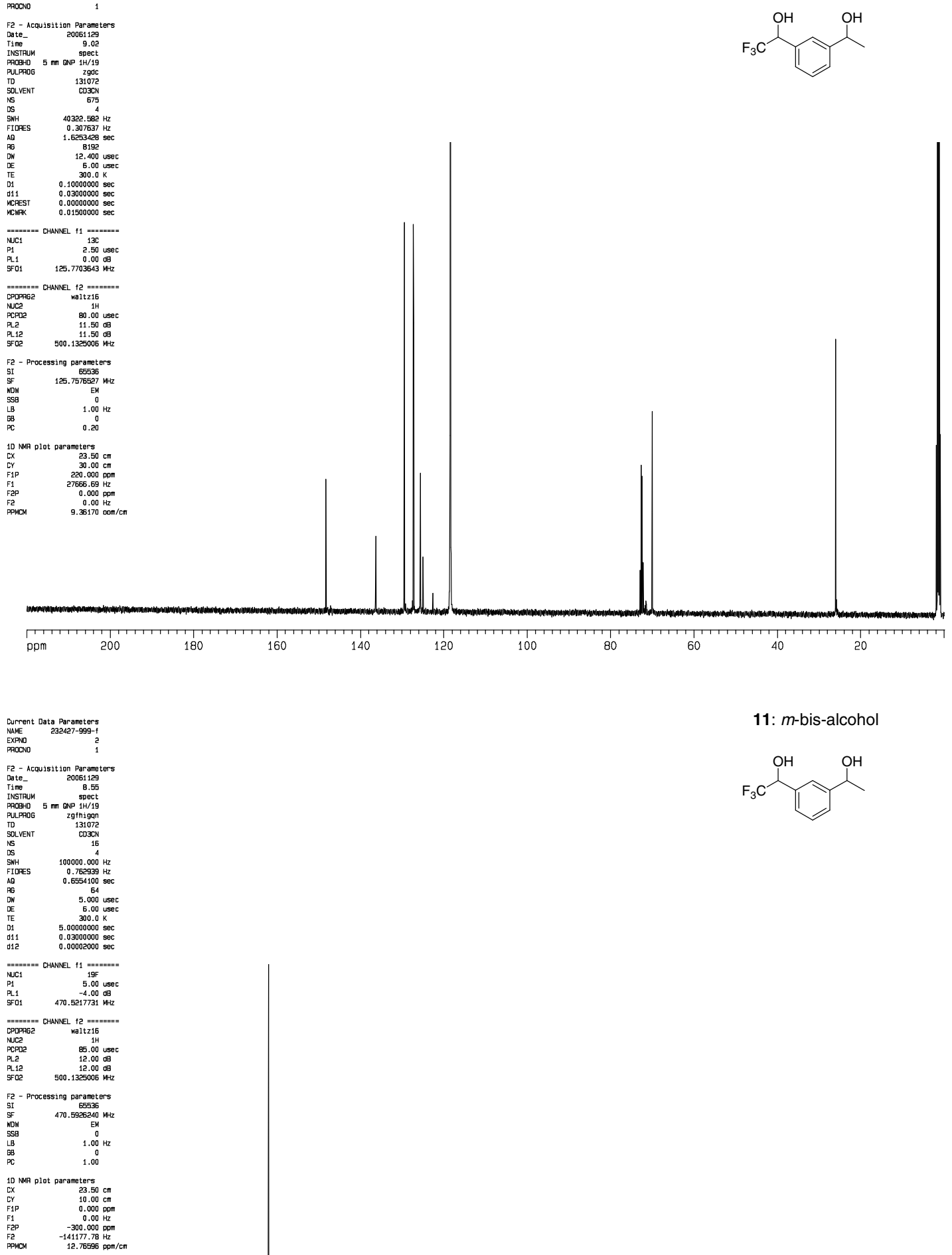

11: $m$-bis-alcohol
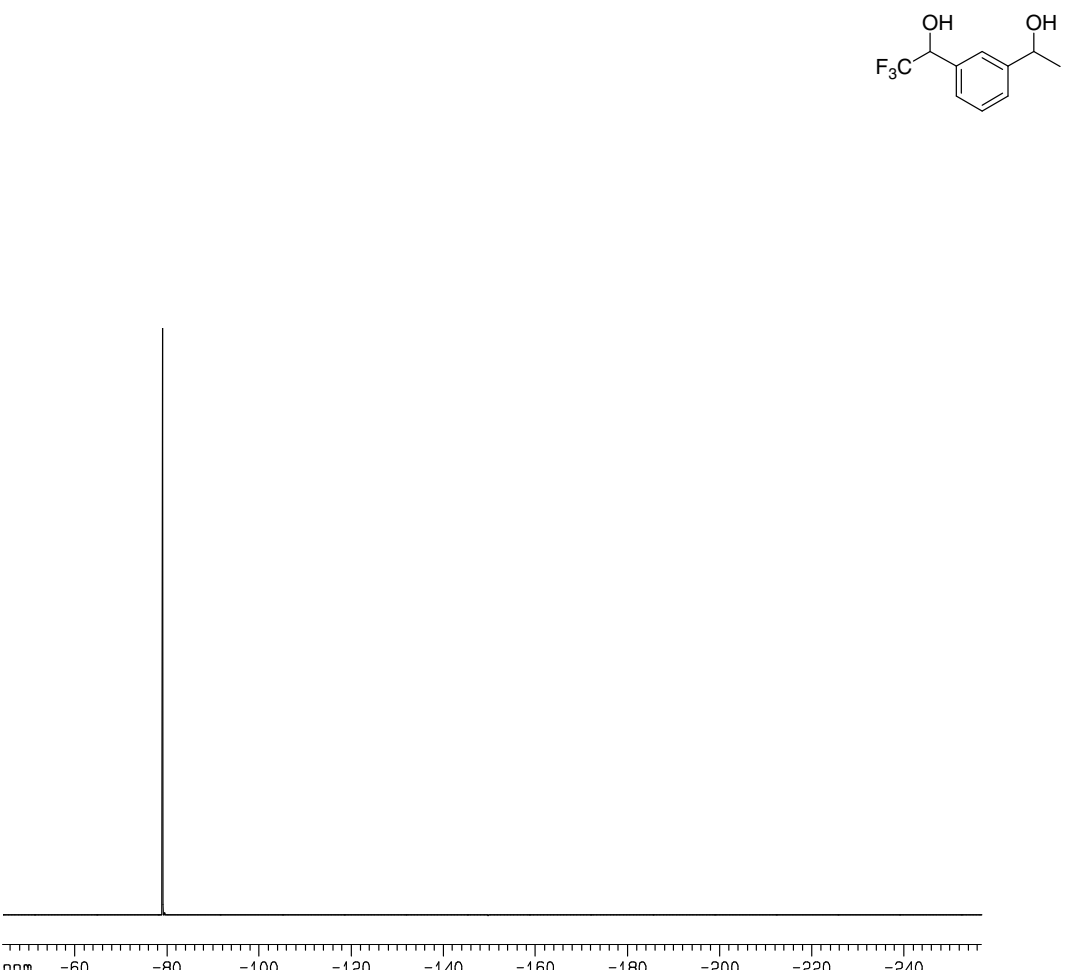
Achiral GC Method:

20. GC000465 (online): Data Analysis [BRENDAN GRAU]

File Graphics Integration Calibration Report Batch Chemstore Miew Abort Help

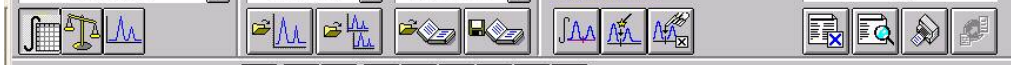

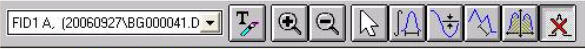

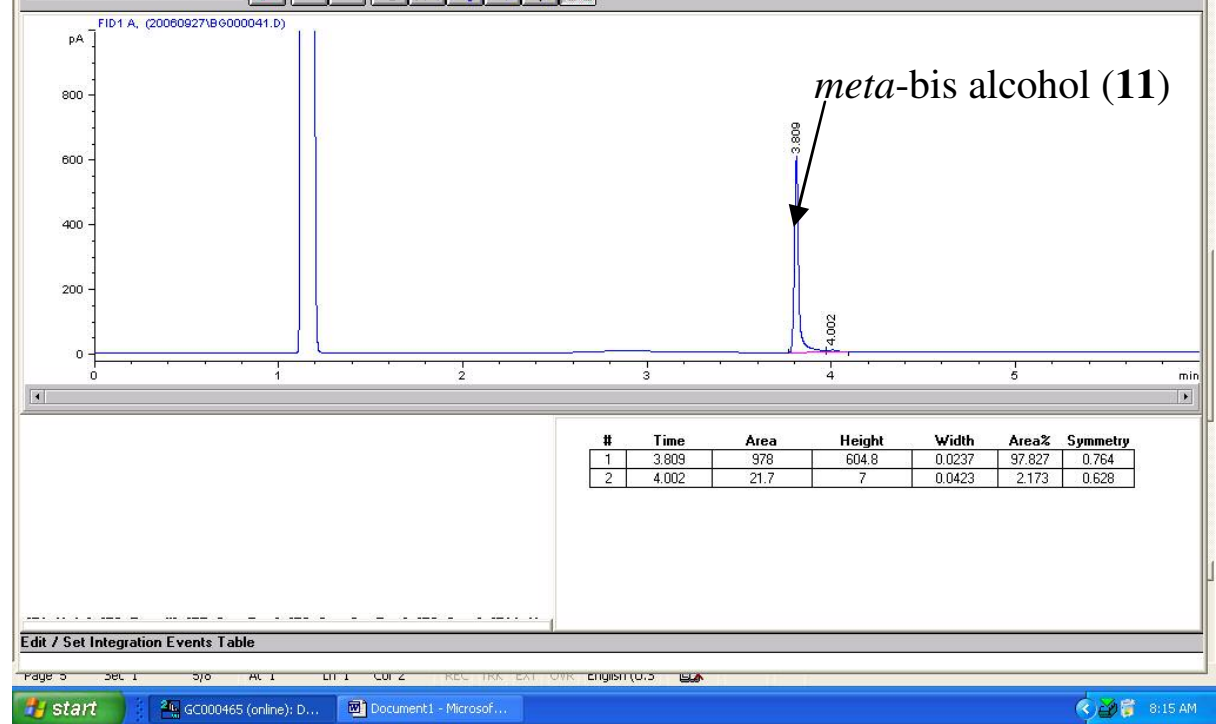

